\section{Structure of the hexameric fungal plasma membrane proton pump in its autoinhibited state}

\author{
Sabine Heit ${ }^{1}$, Maxwell M. G. Geurts ${ }^{1}$, Bonnie J. Murphy ${ }^{2}$, Robin A. Corey ${ }^{1}$, Deryck J. Mills ${ }^{2}{ }$, \\ Werner Kühlbrandt ${ }^{2}$, Maike Bublitz ${ }^{1 *}$
}

\begin{abstract}
The fungal plasma membrane $\mathrm{H}^{+}$-ATPase Pma1 is a vital enzyme, generating a proton-motive force that drives the import of essential nutrients. Autoinhibited Pma1 hexamers in the plasma membrane of starving fungi are activated by glucose signaling and subsequent phosphorylation of the autoinhibitory domain. As related P-type adenosine triphosphatases (ATPases) are not known to oligomerize, the physiological relevance of Pma1 hexamers remained unknown. We have determined the structure of hexameric Pma1 from Neurospora crassa by electron cryo-microscopy at 3.3-Å resolution, elucidating the molecular basis for hexamer formation and autoinhibition and providing a basis for structure-based drug development. Coarse-grained molecular dynamics simulations in a lipid bilayer suggest lipid-mediated contacts between monomers and a substantial protein-induced membrane deformation that could act as a proton-attracting funnel.
\end{abstract}

\section{INTRODUCTION}

The plasma membrane of fungal and plant cells contains a protonpumping P-type adenosine triphosphatase (ATPase) that maintains the intracellular $\mathrm{pH}$ and generates a proton-motive force, driving the import of nutrients by secondary transporters (1). The membrane potential generated by the fungal proton pump Pmal can reach several hundreds of millivolts (2), which requires tight coupling of ATP hydrolysis to strictly unidirectional proton transport.

Considerable effort has gone into characterizing Pma1 [reviewed in (1)], not least because the enzyme has been identified as a potentially valuable drug target against severe invasive mycoses with high mortality rates, particularly in immunocompromised patients $(3,4)$. While there is a large body of biochemical data available, an 8 - $\AA$ map from two-dimensional (2D) electron crystallography has been the highest-resolution structural information available for Pma1 in the past two decades (5), severely hampering any structure-based drug development.

Unlike any other known P-type ATPase, Pmal is a hexamer that localizes to specific ordered microdomains in the fungal plasma membrane (6). It is an abundant membrane protein, and the hexamers can form tightly packed, paracrystalline arrays in starving or stressed cells, as revealed by early electron microscopy (EM) studies $(7,8)$. In addition to the typical P-type ATPase core architecture comprising four domains called $\mathrm{N}$ (nucleotide-binding), $\mathrm{P}$ (phosphorylation), $\mathrm{A}$ (actuator), and M (membrane), Pmal carries sequence extensions at both its $\mathrm{N}$ - and $\mathrm{C}$ termini, which have been implicated in regulation and autoinhibition (fig. S1A) $(9,10)$. The function of the $\mathrm{N}$-terminal extension is still elusive, but the C-terminal autoinhibitory $\mathrm{R}$ domain has been postulated to reduce Pmal activity in starving cells via a clamp-like interaction that immobilizes the cytosolic domains (7). Upon glucose sensing, Pmal is rapidly activated by multiple phosphorylation events in the $\mathrm{R}$ domain.

\footnotetext{
'Department of Biochemistry, University of Oxford, South Parks Road, Oxford OX1 3QU, UK. ${ }^{2}$ Max Planck Institute of Biophysics, Max-von-Laue-Str.3, 60438 Frankfurt am Main, Germany.

*Corresponding author. Email: maike.bublitz@bioch.ox.ac.uk tDeceased.
}

The physiological role of Pma1 hexamers is not yet understood, but in vivo studies in yeast have demonstrated that hexamer formation depends on association with lipids already in the endoplasmic reticulum, which is a prerequisite for surface transport via the secretory pathway (13-15). In addition to a crucial role of very-longchain sphingolipids in Pmal biosynthesis and membrane targeting (13), it has also been shown that Pmal activity in vitro depends strongly on the presence of anionic lipids $(14,15)$. However, structural evidence to understand these findings has been lacking.

We collected single-particle cryo-EM data of the hexameric form of Pmal from Neurospora crassa plasma membrane in its autoinhibited state and obtained a hexamer map at 3.28-Å resolution. An improved map at 3.21- $\AA$ resolution for the Pmal monomer was derived from the same data by symmetry expansion of the hexameric particles followed by focused 3D classification and refinement using a monomer mask (table S1 and fig. S2). An atomic model was built into the monomer map (Fig. 1A) and then extended into a hexameric model by symmetry-assisted placement of five additional copies into the hexamer map (Fig. 1B). The structure reveals the basis for proton transport, autoinhibition, and hexamer formation. Coarse-grained molecular dynamics (MD) simulations in a nativelike membrane indicate an accumulation of specific anionic lipids at the monomer interface. A concomitant protein-induced deformation of the lipid bilayer in this region appears to form a cationattracting funnel and reduce the membrane thickness in the vicinity of the proton-translocating residues. The Pmal structure is a major advance toward the development of specific inhibitors, and our findings further highlight the important role of lipids in Pmal function.

\section{RESULTS}

The structure of Pma 1

The Pma1 monomer has a typical P-type ATPase fold, with four conserved domains: the $\mathrm{M}$ domain comprising the 10 membranespanning $\alpha$ helices M1 to M10, and the 3 cytosolic domains A, N, and P (Fig. 1A and fig. S1A). The additional C-terminal autoinhibitory $\mathrm{R}$ domain forms an $\alpha$ helix that folds against the $\mathrm{P}$ domain. Our molecular model comprises $>90 \%$ of all residues, lacking only the $\mathrm{N}$-terminal extension (residues 1 to 65), and two loops (A-M3, 
A

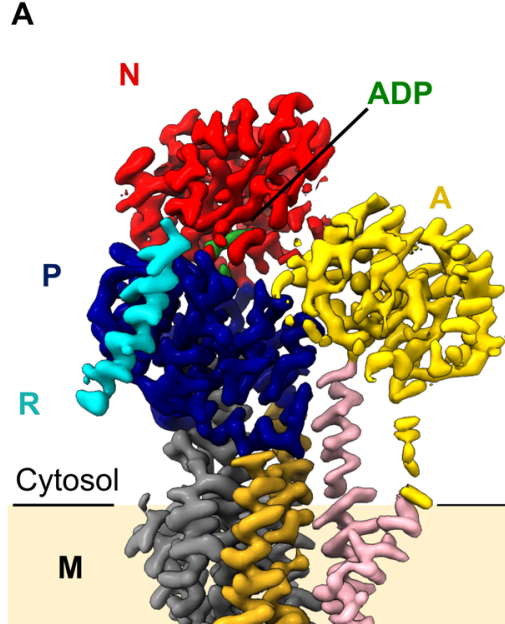

B
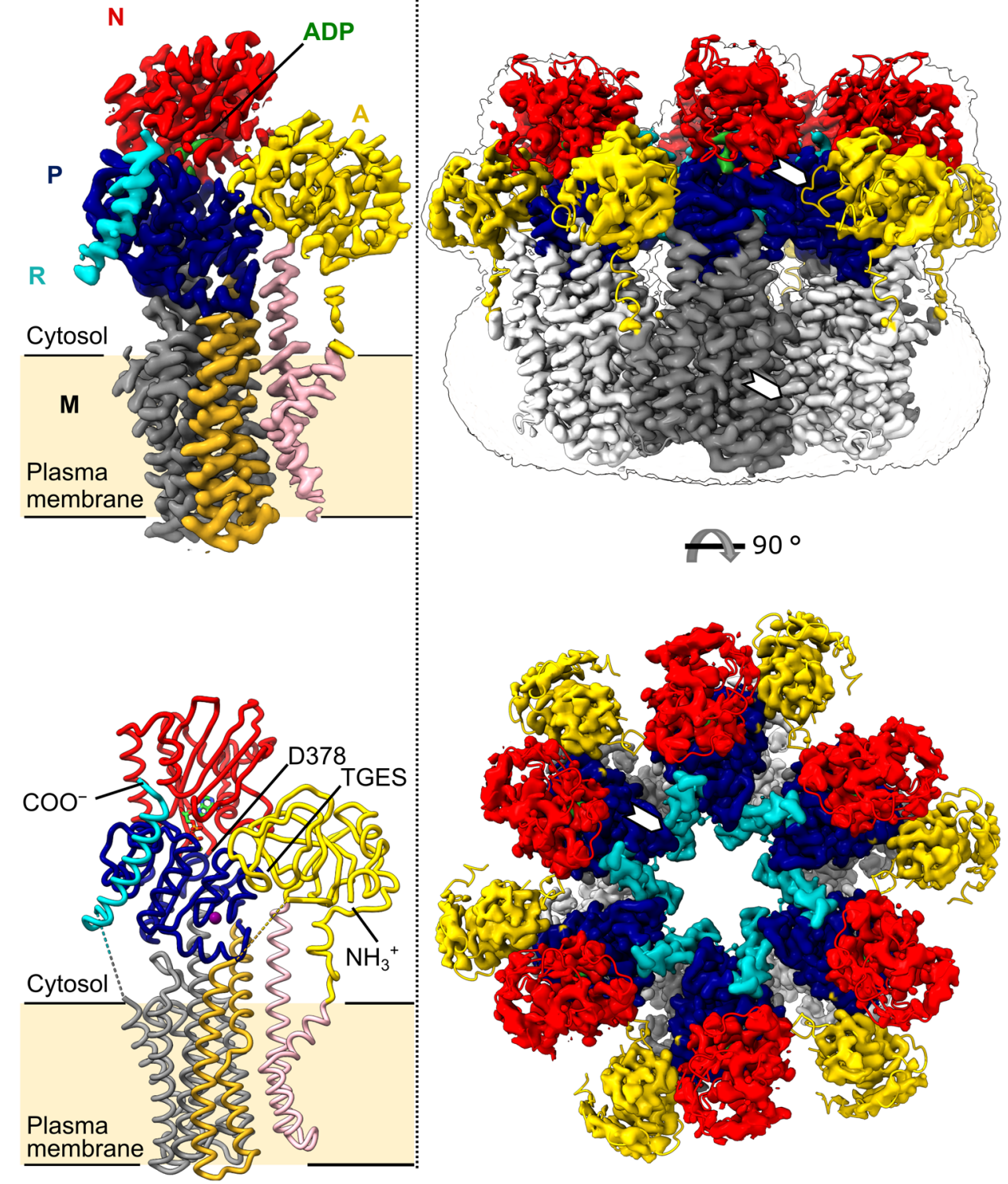

Fig. 1. The cryo-EM structure of monomeric and hexameric Pma1. (A) Cryo-EM map (top) and structural model (bottom) of the Pma1 monomer subunit. Nucleotidebinding $(N)$ domain in red, actuator $(A)$ domain in yellow, phosphorylation $(P)$ domain in blue, regulatory (R) domain in cyan, and adenosine diphosphate (ADP) in green The 10 helices of the M domain are colored in pink (M1-M2), gold (M3-M4), and gray (M5-M10). In the bottom panel, ADP is shown as sticks and a potassium ion as a purple sphere; unmodeled loops are indicated by dashed lines. (B) Overlay of cryo-EM map and structural model of the Pma1 hexamer in side view (above; with map outline delineating the detergent micelle) and top view (below). The $\mathrm{M}$ domains of individual monomers are shown in alternating shades of gray, and cytosolic domains and ADP are colored as in (A). White arrows indicate contact sites between monomers.

residues 269 to 283 , and M10-R, residues 881 to 891 ), for which no continuous density was visible in the map, probably because of flexibility.

The Pmal hexamer (Fig. 1B) forms a ring with sixfold symmetry, with a maximum outer diameter of $167 \AA$ and an inner cavity measuring 24 to $55 \AA$ in diameter in the cytosolic and transmembrane regions, respectively. Monomer contacts are mediated by both the cytosolic domains and the transmembrane domains. Specifically, there are two connections between the cytosolic domains: a loose contact between the conserved Thr-Gly-Glu-Ser (TGES) loop in the
A domain (residues 231 to 235) and two $\alpha$-helix termini in the $P$ domain $\left(\mathrm{Tyr}^{541}, \mathrm{Arg}^{570}\right.$, and $\mathrm{Gl}^{571}$ ) (Figs. 1B and 2A). The second, more extensive contact between the $\mathrm{P}$ domains of adjacent monomers is mediated entirely via the autoinhibitory $\mathrm{R}$ domain (Fig. 1B, table S2, and see further discussion below). The interactions in the transmembrane region involve residues in M3 of one monomer, and M7 and M10 of the adjacent monomer, augmented by two polar contacts from Ser ${ }^{316}$ and $\mathrm{Asn}^{317}$ in L3-L4 to Gln ${ }^{780}$ (M7) and $\mathrm{Arg}^{859}$ (M10) (Figs. 1B and 2B and table S3). Most hydrophobic residues involved in the hexamer contact are functionally conserved. 
A
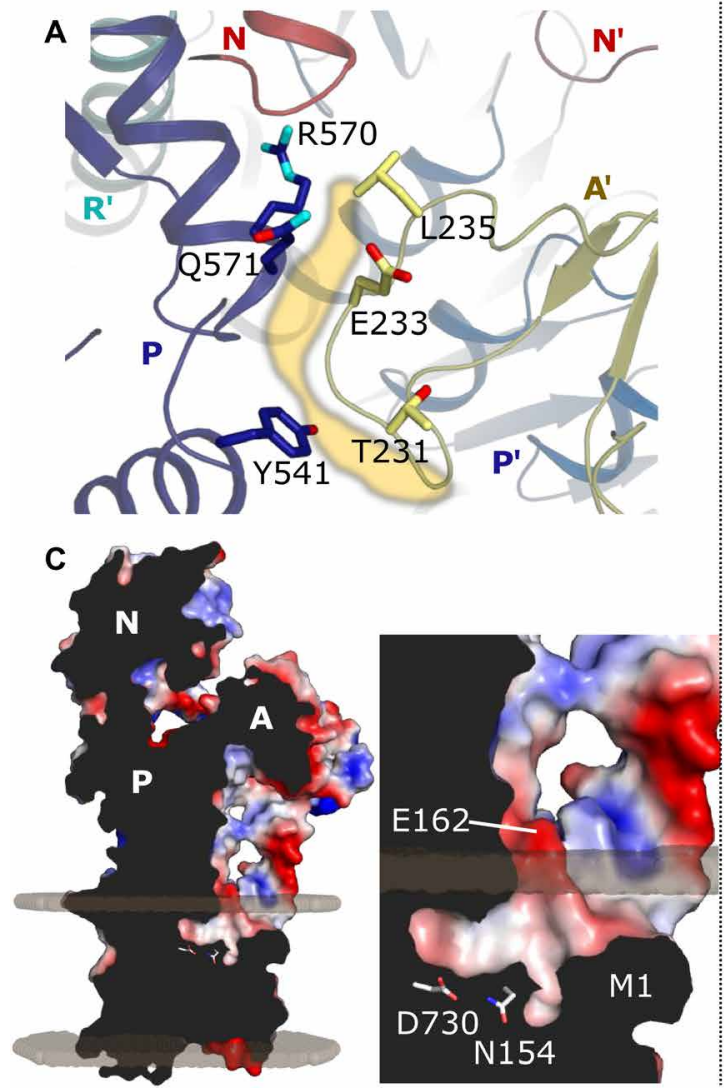

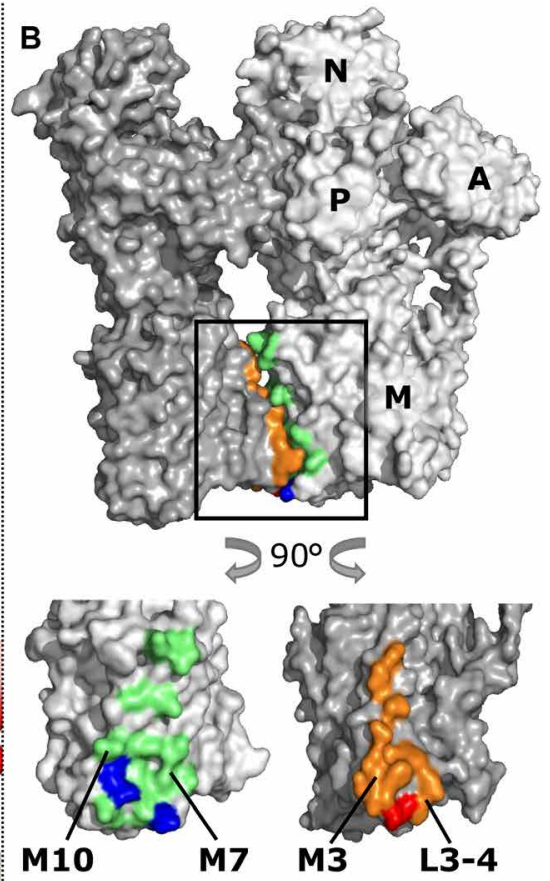

M10

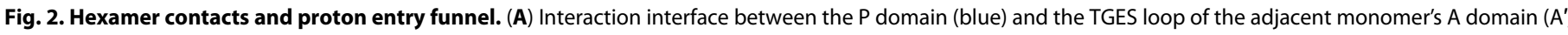

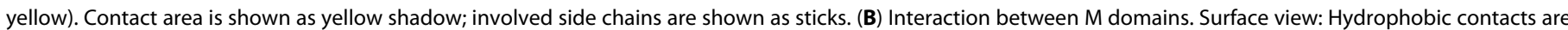

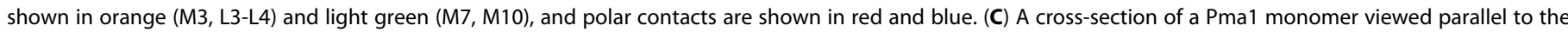

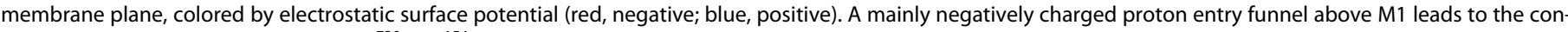
served proton acceptor residue pair Asp ${ }^{730} / \mathrm{Asn}^{154}$ (shown as sticks). The funnel extends below the predicted lipid bilayer boundary (45) shown as shaded spheres.

P-type ATPases go through a conformational cycle described by the E1/E2 (also known as Post-Albers) scheme (fig. S1B), with E1 denoting states with a high affinity to the cytosolic substrate ion $\left(\mathrm{H}^{+}\right.$in the case of Pma1). The domains of Pmal are arranged in an E1 conformation, with the $\mathrm{N}$ domain in proximity to the $\mathrm{P}$ domain, as required for MgATP-mediated phosphorylation of Asp ${ }^{378}$. The observed state resembles the $\beta, \gamma$-methyleneadenosine $5^{\prime}$-triphosphate (AMPPCP)bound $E 1$ structure of the plant proton pump AHA2 (16) and the pre-ion binding, open-to-inside state of SERCA (MgE1) (3.0 and $3.5 \AA$ root mean square deviation over all $\mathrm{C} \alpha$ atoms, respectively; fig. S3) (17). The conserved nucleotide binding pocket is occupied by a MgADP molecule (fig. S4A). We added $600 \mu \mathrm{M} \mathrm{AlF} x$ to the sample before grid preparation, with the aim to stabilize the enzyme in its transition state of phosphorylation, E1 P. However, we did not find a clear density for $\mathrm{AlF}_{x}$ in its expected position between the adenosine diphosphate (ADP) $\beta$-phosphate and the Asp ${ }^{378}$ carboxyl group. Weaker map features in this region match possible positions of a second $\mathrm{Mg}^{2+}$ ion or might indicate a low occupancy of $\mathrm{AlF}_{x}$. This finding reflects the reduced affinity of autoinhibited, basal state Pmal to $\mathrm{AlF}_{x}$ compared to the active form (18). We have hence captured the basal state before ATP hydrolysis, occupied by excess MgADP.

As expected for a prephosphorylation $E 1$ state, access from the cytosol to the proton-binding site is open in our structure. The M1 helix with its characteristic $90^{\circ}$ kink (induced by Pro $^{123}$ ) is embedded deeply inside the membrane, forming a wide funnel-shaped access toward the $\mathrm{H}^{+}$binding site in $\mathrm{M} 6$, similar to the $\mathrm{Ca}^{2+}$ entry funnel in SERCA (Fig. 2C, and see more detailed discussion below) (17). The mouth of this access pathway is negatively charged because of the conserved $\mathrm{Glu}^{162}$ in $\mathrm{M} 2$ (Fig. 2C), and it extends into a large aqueous cavity between M1, M2, M6, and an unwound region in M4 around the conserved Pro ${ }^{335}$ (Fig. 3). The cavity is almost twice as large $\left(\sim 746 \AA^{3}\right)$ as in the plant proton pump $\left(\sim 380 \AA^{3}\right)(16)$ and can accommodate up to 18 water molecules. While the horizontal, $\mathrm{N}$-terminal part of M1 is amphipathic in SERCA, it is strongly hydrophobic in Pma1, except for Lys ${ }^{115}$, which points upward toward the aqueous cytosolic phase, as a typical "snorkeling" residue (Fig. 3A, inset). The C-terminal part of M1 contains two hydrophilic residues: Gln ${ }^{125}$ at the upper end, pointing into the interior of the protein, and $\mathrm{Glu}^{129}$ at the lower end, contacting the membrane lipid environment. In line with its accessibility from the hydrophobic membrane center, Glu $^{129}$ has been identified as the site of covalent dicyclohexylcarbodiimide (DCCD) binding and inhibition of Pmal (19). The structure reveals that DCCD might lock M1 in this open conformation, hence preventing proton occlusion and transport. While the physiological role of $\mathrm{Glu}^{129}$ is currently unknown, it is conserved throughout plasma membrane $\mathrm{H}^{+}$- and $\mathrm{Ca}^{2+}$-pumping P-type ATPases. 

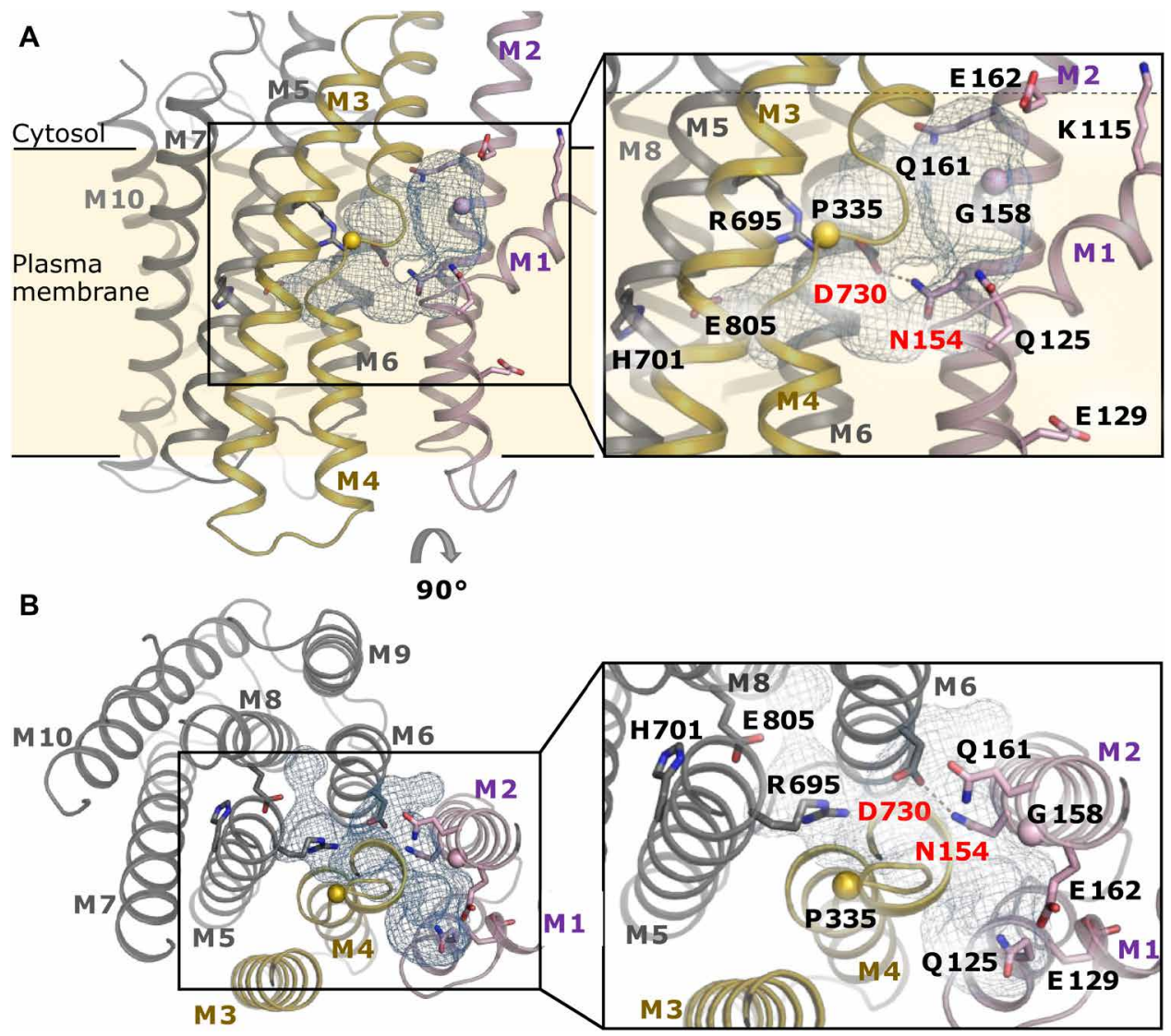

Fig. 3. Intramembranous cavity and proton-binding site of Pma1. (A) Aqueous cavity between M1, M2, M6, and an unwound region of M4 (the conserved Pro ${ }^{335}$ is shown as a sphere). Important residues for proton transport and the snorkeling K115 (top right corner) are shown as sticks (sphere for glycine). M1-M2 are colored pink, M3-M4 are colored gold, and M5-M10 are colored gray. In the enlarged view, the putative hydrogen bond (2.9 $\AA$ ) between the proton acceptor/donor Asp ${ }^{730}$ and Asn ${ }^{154}$ (labeled in red) is shown as dashed line. (B) $\mathrm{Arg}^{695}$ is facing the cavity and could form a salt bridge with $\mathrm{Asp}^{730}$ (labeled in red) after proton release. The conserved residues $\mathrm{His}^{701}$ and $\mathrm{Glu}^{805}$, which are both necessary for proton pumping, interact with each other in the $E 1$ state.

The proton-acceptor/donor in Pmal is the conserved aspartate residue Asp ${ }^{730}$ in M6 (Fig. 3). This residue corresponds to Asp ${ }^{684}$ in AHA2 and the $\mathrm{Ca}^{2+}$-coordinating residue $\mathrm{Asp}^{800}$ in SERCA and has been shown to be essential for proton transport and $E 1 \rightarrow E 2$ transitions in Pmal $(20,21)$. Asp ${ }^{730}$ is situated adjacent to the internal cavity and opposite the equally highly conserved $\mathrm{Asn}^{154}$ in M2, at a distance suitable for capturing a proton within a hydrogen bond (2.9 ̊̊ between the aspartate carboxyl and the asparagine amide). Proton release to the extracellular side has been suggested to be driven by a conformational change that breaks this hydrogen bond and instead allows the formation of a salt bridge between the deprotonated aspartate and an adjacent arginine residue $\left(\mathrm{Arg}^{655}\right.$ in the plant $\mathrm{H}^{+}$-ATPase AHA2) while concomitantly opening up the exit pathway between M1, M4, and M6 $(16,22)$. The arginine has also been suggested to act as a positively charged "gatekeeper" preventing proton reentry into the cavity. In Pmal, the equivalent of $\mathrm{Arg}^{655}$ in AHA2 is $\mathrm{His}^{701}$ in M7, which would not be able to fulfill this role - a fact that has raised doubts about the similarity of the mechanisms of the two proton pumps. In our structure, the side chain of $\mathrm{His}^{701}$ points away from the proton-binding site, interacting with $\mathrm{Glu}^{805}$ in M8 (Fig. 3). The precise role of this residue pair is currently unclear, but both residues are necessary for proton pumping in Pmal (23). The key functional role postulated for $\mathrm{Arg}^{655}$ in
AHA2 is likely taken over by $\mathrm{Arg}^{695}$ in Pma1, which is strictly conserved in fungi and positioned 2.5 helix turns up from $\mathrm{His}^{701}$, facing the ion-binding site (Fig. 3, A and B).

The C-terminal autoinhibitory $\mathrm{R}$ domain extends from the cytosolic end of M10 into a brief region of disorder and then forms a mainly $\alpha$-helical structure that lies adjacent to the P domain (Fig. 4A), with which it forms several polar interactions as well as a surprisingly large number of hydrophobic contacts, also involving residues in the $\mathrm{P}$ domain of the adjacent monomer (Fig. 4B). The distance across the unmodeled gap between the $\mathrm{C}$ terminus of M10 and the $\mathrm{N}$ termini of two adjacent $\mathrm{R}$ helices is very similar, preventing an unambiguous assignment of $\mathrm{R}$ domains to their respective monomers. The current assignment is based on a short extension at the $\mathrm{N}$ terminus of the R helix that points toward one of the two M10 termini (fig. S4B).

\section{Local lipid accumulation and protein-induced membrane deformation}

To probe Pma1-lipid interactions in a native-like bilayer, we ran coarse-grained MD simulations of Pma1 in a complex bilayer of different lipid types occurring in $N$. crassa (fig. S5A and table S6) $(13,24-26)$. To prevent any positional bias of lipids placed inside the ring, one monomer was omitted from the hexamer to allow a 
A

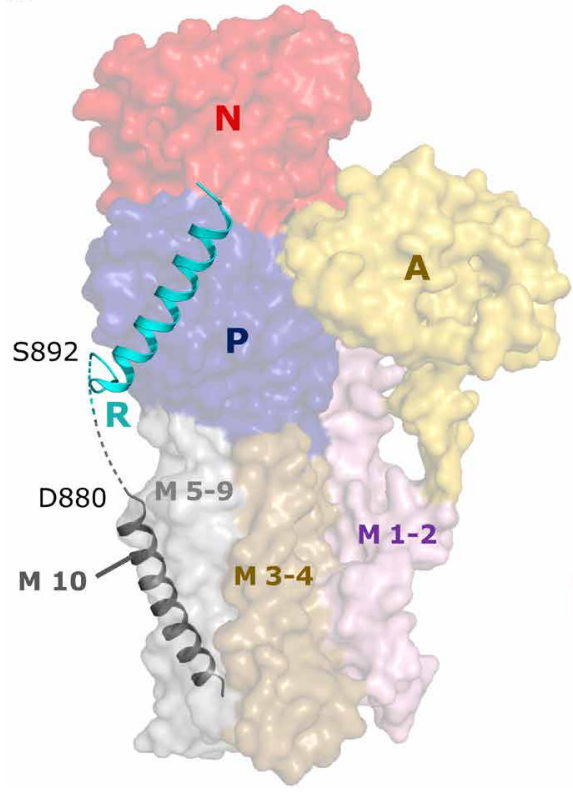

B

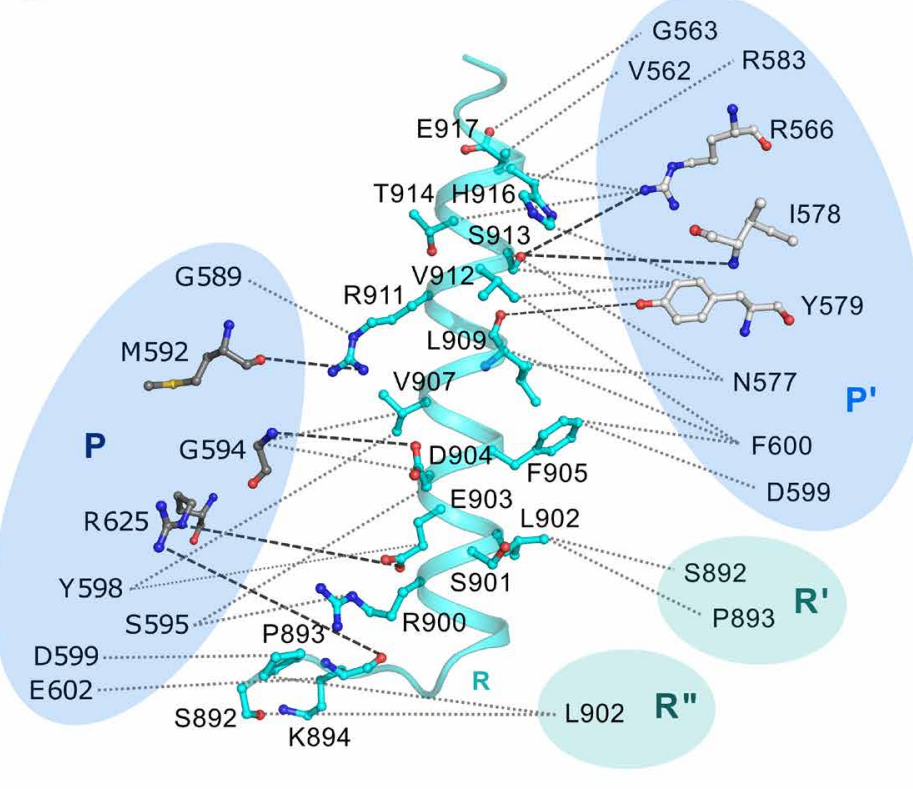

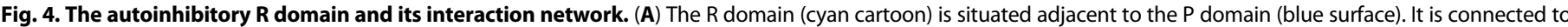

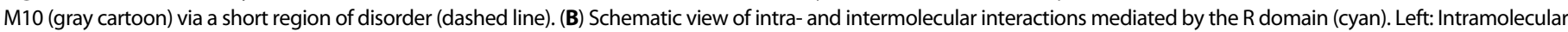

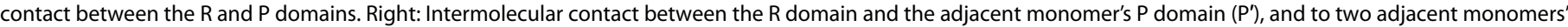

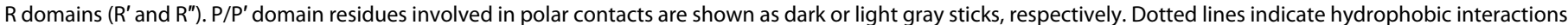

free lipid exchange during the simulations. Fractional interaction times of each lipid at the Pmal surface suggest two putative binding sites at the monomer interface: site I in the inner membrane leaflet (involving $\mathrm{M} 1, \mathrm{M} 3$, and $\mathrm{M} 10^{\prime}$ ) and site II in the outer membrane leaflet (between M4 and M10') (Fig. 5A), with a preferred binding of phosphatidylserine (PS) and phosphatidylcholine (PC), respectively (Fig. 5B and fig. S6). Our cryo-EM maps do not clearly resolve any tightly bound lipids, probably due to a dynamic and inhomogeneous nature of lipid binding. Lipid-mediated hexamer contacts are in line with the observations that hexamer formation in vivo depends on an intact lipid biosynthesis pathway (11-13). In addition, the hexamer is sensitive to treatment with detergents such as octyl glucose neopentyl glycol (OGNG) in vitro (fig. S7). In our coarse-grained simulations, both sites preferably interact with the double-unsaturated 16:2/18:2 species, as compared to the monounsaturated 16:0/18:2 (Fig. 5B and figs. S6, S8, and S9). A local lipid density analysis shows that the accumulation of 16:2/18:2 PS (DIPS) at site I is accompanied by an extended clustering of this lipid in the vicinity (Fig. 5C). 16:2/18:2 PC (DIPC) is the only other lipid that also accumulates at the interface (at site II), albeit to a lesser extent than DIPS, and has an otherwise even distribution around the protein (Fig. 5C and fig. S9). Accumulation could be driven either by localized clustering of lipids with unsaturated tails, which would explain the presence of both DIPS and DIPC, or instead by clustering of the acidic PS head groups, which has been observed previously (27). A preferential occupation of site I with DIPS is in line with the fact that ATPase activity of detergentpurified Pmal in vitro depends on the addition of acidic phospholipids (14). A very recent study also found an approximate threefold enrichment of PS in styrene-maleic acid (SMA)-solubilized Pma1 compared to the average plasma membrane composition (26).

The simulations further suggest that the Pmal protein has a considerable impact on the local membrane structure: Near the M1-M4 bundle, the inner membrane leaflet is locally thinned, a "dip" that is most pronounced near the unwound region of M4 and along the outside-facing surfaces of M6 (the helix containing the protonaccepting $\mathrm{Asp}^{730}$ ) and M9 (Fig. 5D and fig. S10). Opposite this dip, on the inside of the ring, the cytosolic membrane surface bulges upward toward the $\mathrm{P}$ domain, probably attracted by the partial positive charge at the N-terminal end of M3 and by Lys ${ }^{749}$ in the L6-L7 loop. Both the "dip" and the "bulge" are also evident from the cryo-EM map of the micelle surrounding the Pmal hexamer particles [likely consisting of a mixture of $\beta$-dodecyl-maltopyranoside (DDM) with residual membrane lipids] (Fig. 5E). Such a "chute"-like arrangement of negatively charged lipid headgroups near the mouth (also negatively charged; see Fig. 2C) of the ion entry funnel could facilitate the attraction of protons from the cytosol into the binding site. In support of this hypothesis, the simulations show an increased local density of $\mathrm{Na}^{+}$ions from the solvent in this region (Fig. $5 \mathrm{~F}$ ).

The extracytosolic leaflet plug inside the hexamer is shifted upward in the simulations - an effect of unknown cause that has also been observed by atomic force microscopy inside the rotor rings of ATP synthase (28). This effect is, however, not evident from the micelle shape, in contrast to the cytosolic leaflet deformations. Together, the simulations suggest an approximate 10 - $\AA$ decrease in membrane thickness (from $\sim 37$ to $\sim 27 \AA$ ) in the region where the protein is inserted and a local thickening to $\sim 54 \AA$ in the membrane region below the P domain (Fig. 5D and fig. S10).

\section{Model for proton pumping and autoinhibition}

To develop a model for the proton pumping cycle of Pmal (fig. S11), we generated a series of homology models based on SERCA in the transition of phosphorylation $(E 1 \sim \mathrm{P})$, open-to-outside $(E 2 \mathrm{P})$, transition of dephosphorylation (E2 P), and dephosphorylated (E2) states. The transition state of phosphorylation, $E 1 \sim \mathrm{P}$, is linked to ion occlusion 
A

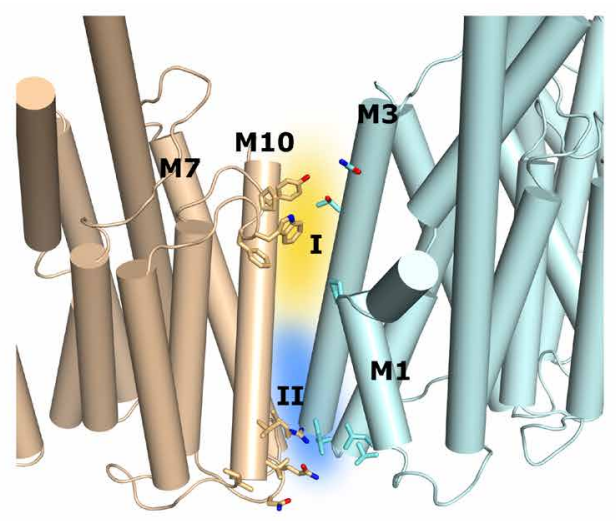

C

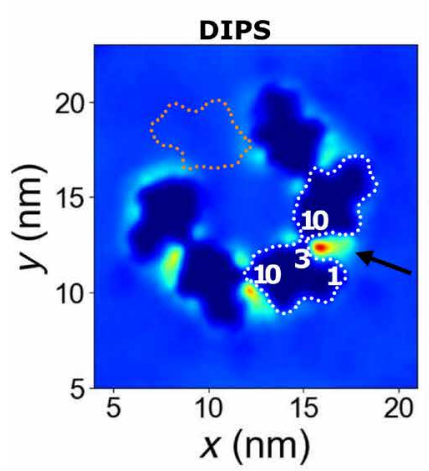

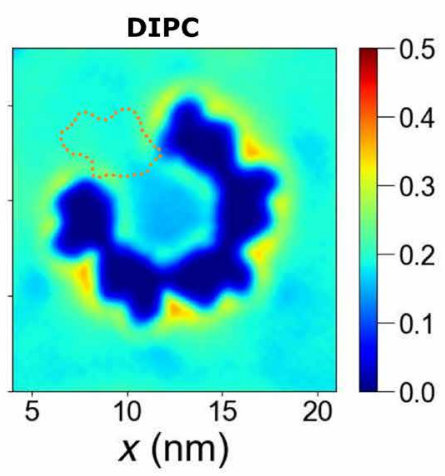

site I

site II
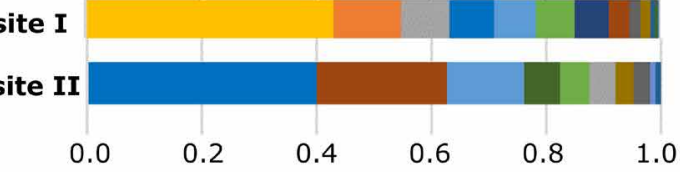

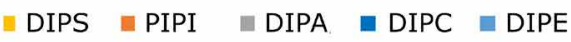

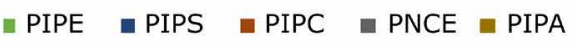

DPCE $\square$ PVSM $=\mathrm{XNSM}$

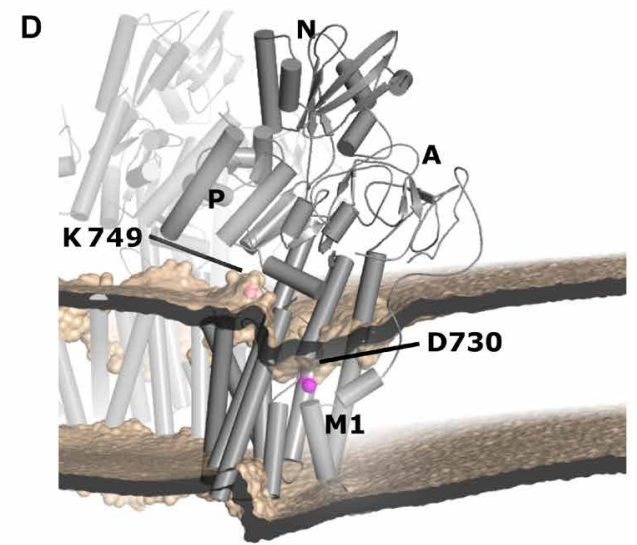

E

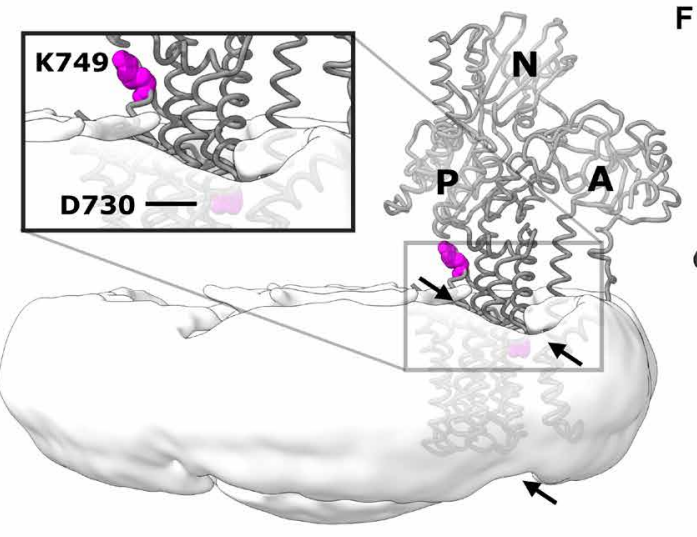

$\mathbf{F}$

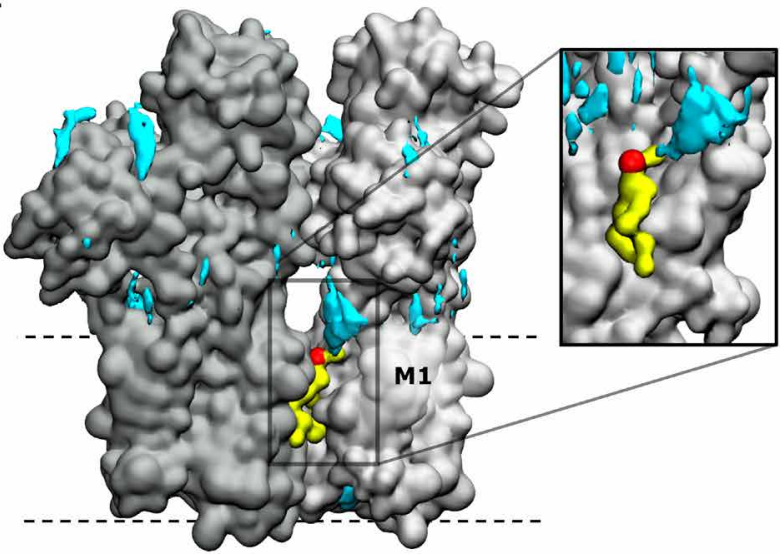

Fig. 5. Lipid interactions of Pma1. (A) Putative lipid binding sites (I and II) at the monomer interface, suggested by MD simulations. (B) Fractional interaction times of lipids in sites I and II, defined as the number of simulation frames in which a lipid is within $0.6 \mathrm{~nm}$ of a given site. (C) Simulated lipid densities for DIPS and DIPC. Arrow in DIPS points at the pool clustering outside the monomer interfaces. Values correspond to average number of molecules per cubic nanometer and do not account for the respective membrane composition fraction. White dotted lines and numbers indicate monomer outlines and transmembrane helix positions, respectively. Orange dotted lines indicate the omitted sixth monomer. (D) Side view of the coarse-grained bilayer deformation at the monomer interface. The bilayer (wheat) is shown as the average phosphate position across five simulation repeats. An atomistic Pma1 model was aligned for analysis. (E) Cryo-EM map of hexameric Pma1, filtered to $12 \AA$, showing only the micelle after masking the protein map in Coot (14). The C $\alpha$ trace of one monomer is shown for reference, with the side chains of D730 and K749 highlighted in magenta. The micelle is locally distorted near the monomer interface (arrows). (F) Locally increased density of $\mathrm{Na}^{+}$ions (cyan) around the monomer interface. A representative DIPS molecule in site I is shown in yellow, with the phosphate group highlighted in red.

within the binding site $(30,31)$. In this state, $\mathrm{Asp}^{730}$ moves away from Asn ${ }^{154}$, implying a break of the suggested hydrogen bond (Fig. 3B). Nevertheless, the predicted $\mathrm{p} K_{\mathrm{a}}$ (where $K_{\mathrm{a}}$ is the acid dissociation constant) value of $\mathrm{Asp}^{730}$ in this state $(7.2)(32,33)$ suggests that it is protonated, likely because it is buried inside the hydrophobic core of the protein. Two carbonyl oxygens on the backbones of $\mathrm{Il}^{332}$ and $\mathrm{Ala}^{729}$ may act as stabilizing H-bond acceptors. $\mathrm{Arg}^{695}$, the conserved residue also suggested to be involved in proton repulsion in $E 2$, takes a position that shields Asp ${ }^{730}$ from the cytosolic side as the entry channel closes and possibly prevents the acquired proton from escaping back to the cytosol.

In the open-to-outside E2P homology model, Asp ${ }^{730}$ lies at the inner end of the exit funnel, which is lined by predominantly hydrophobic 
side chains, except for $\mathrm{Asp}^{143}$ approximately halfway down the funnel. Both $\mathrm{His}^{701}$ and $\mathrm{Arg}^{695}$ are in the vicinity of $\mathrm{Asp}^{730}$, and the $\mathrm{Arg}^{695}$ side chain can freely rotate into bonding distance (fig. S12). It could hence prevent reprotonation of $\mathrm{Asp}^{730}$ before the exit pathway is fully closed, working as an in-built counterion to neutralize Asp ${ }^{730}$ during the $E 2 \rightarrow E 1$ transition, as suggested for AHA2 $(16,22)$. Functional studies suggest that Pmal has a very low affinity for inorganic phosphate or phosphate mimics such as $\mathrm{MgF}_{x}^{-}$(18), indicating a rapid release and thus also a short lifetime of the extracellular proton exit gate. There is a conspicuous cluster of negatively charged residues $\mathrm{Glu}^{139}, \mathrm{Asp}^{140}$ (L1-L2), Asp ${ }^{143}$ (M2), Glu ${ }^{324}$ (M4), and Glu ${ }^{720}$ (M6) at the extracellular end of the funnel (fig. S12), which would facilitate proton extrusion.

Autoinhibition of Pmal by the R domain has been postulated to function independent of the oligomeric state via a cis-acting immobilization of the cytosolic domains. This is supported by the fact that an excess of free R peptide counteracts autoinhibition (10). In our structure, the R helix interacts exclusively with the $\mathrm{P}$ domain, which is expected to move relatively little during proton pumping. All our (monomeric) modeled conformations can accommodate the $\mathrm{R}$ helix in this position without any clashes, which is in line with the observation that binding of free $\mathrm{R}$ peptide does not interfere with activity. However, the distance between the C terminus of M10 and the $\mathrm{N}$ terminus of the $\mathrm{R}$ helix increases from $30 \AA$ in the $E 1$ structure to $41 \AA$ in the $E 2$ homology model (fig. S13A), supporting the "clamping" model of autoinhibition, as the 16-residue linker between $\mathrm{M} 10$ and the R helix might restrain the $E 1 \rightarrow E 2$ transition.

In Saccharomyces cerevisiae, release of the autoinhibition upon glucose sensing has been linked to phosphorylation of $\mathrm{Ser}^{899}\left(\mathrm{Ser}^{901}\right.$ in N. crassa) by Ptk2 (34) and tandem phosphorylation of $\mathrm{Ser}^{911} / \mathrm{Thr}^{912}$ (Ser ${ }^{913} / \mathrm{Thr}^{914}$ in N. crassa) by an unknown kinase (35). Ser ${ }^{899}$-P leads to an increase in $K_{\mathrm{m}}$ (Michaelis constant), and $\mathrm{Ser}^{911}-\mathrm{P} / \mathrm{Thr}^{912}-\mathrm{P}$ to an increase in $V_{\max }(9,24-36)$. Notably, neither of these residues is located at the binding interface of the cis-acting R domain (Fig. 4). A release of the $\mathrm{R}$ domain from the $\mathrm{P}$ domain in a monomeric context would therefore need to involve phosphorylation-induced structural alterations within the $\mathrm{R}$ helix itself, leading to disruption of $\mathrm{R}-\mathrm{P}$ interactions. While such a mechanism is not impossible, it seems unlikely, which would point toward a role for the hexamer as the physiologically relevant unit of Pmal.

\section{The role of the hexamer}

Originally, the hexamer has been suggested to serve as a spacesaving form for efficient trafficking and for storage of large amounts of autoinhibited protein in the plasma membrane of starving cells $(10,37)$. Upon glucose sensing, Pmal is rapidly activated by phosphorylation of the $\mathrm{R}$ domain, which would release it from the $\mathrm{P}$ domain. There is no evidence to suggest that the hexamer needs to come apart for Pmal to become fully active. While monomeric Pma1 is capable of pumping protons (38), Pmal activity in vitro is mostly reported in a hexameric context, and the physiological importance of multimeric plasma membrane $\mathrm{H}^{+}$-ATPases is becoming more and more evident $(39,40)$. Studies with purified plasma membranes have indicated the presence of either two cooperative ATP-binding sites or an even larger number of sites with a lower degree of cooperativity (41). In addition, the stoichiometry of DCCD inhibition suggests that binding of one molecule of DCCD is sufficient to block ATP hydrolysis in 2.5 molecules of Pma1 (19), suggesting that the physiological unit of Pmal is multimeric. Hexameric assemblies of the homology models show that there is sufficient space to accommodate the expected cytosolic domain rearrangements throughout the catalytic cycle, without any clashes (Fig. 6A). The models further suggest either that the hexameric ring would need to expand slightly to accommodate the opening of the extracellular pathway or that Pmal does not open up as widely as SERCA, which served as a structural template. If this is the case, it may reflect the smaller size of the transported ion and the need to ensure quick closure upon proton release to prevent backflow at high membrane potentials.

We investigated all possible combinations of neighboring conformations, assuming an untethered $\mathrm{R}$ domain (see below). None of the 20 combinations clash, indicating that there is no cooperative domain movement as none of the catalytic states of one monomer affects that of its neighbors.

\section{Autoinhibition and activation in the hexamer}

Comparing the autoinhibited hexamer structure with the homologymodeled active state in the process of phosphoryl transfer, the domain packing of the autoinhibited state is tighter. Accordingly, the accessible surface area in the autoinhibited hexamer is at a minimum $\left(213,100 \AA^{2}\right)$, and all homology-modeled hexamers have substantially increased outer diameters compared to the $167 \AA$ of the autoinhibited form (Fig. 6A and fig. S13B), suggesting that the early electron micrographs of dense Pmal arrays [with estimated diameter of $150 \AA$ (8)] represent the autoinhibited form.

The $\mathrm{R}$ domains are closely sandwiched between adjacent $\mathrm{P}$ domains, forming an extensive interaction network (Figs. 1B and 4). R domains of adjacent monomers interact directly: $\mathrm{Ser}^{892}$ and $\mathrm{Pro}^{893}$ interact with $\mathrm{Leu}^{902}$ of the adjacent $\mathrm{R}$ helix, thereby linking all $\mathrm{R}$ domains within the hexamer. During pumping, the $\mathrm{P}$ domains would need to increase their mutual distance, breaking the $\mathrm{R}$ domain-mediated cross-link. Hexamer contacts could hence augment the intramolecular clamping effect of the $\mathrm{R}$ domain. Furthermore, in four of the possible neighboring combinations within the hexamer (E2:E1, E2:E1 P, E2P:E1, and E2 P:E1), the $\mathrm{R}$ helix cannot be accommodated in the position observed in the $E 1$ state. Together, this suggests that either the monomers within the hexamer must move in a coordinated manner or, more likely, the $\mathrm{R}$ domain is displaced from the P domain upon Pmal activation.

Phosphorylation of $\mathrm{Ser}^{913} / \mathrm{Thr}^{914}$ would disturb the contact between the $\mathrm{R}$ domain of one monomer and the adjacent $\mathrm{P}$ domain. In contrast, $\mathrm{Ser}^{901}$ points into the center of the hexameric ring, and hence, its phosphorylation by Ptk2 is unlikely to affect the intermolecular contact directly. A single molecule of Ptk2 can fit into the center of the Pmal hexamer and could lead initially to the phosphorylation of Ser ${ }^{901}$, followed by ATP binding and phosphoryl transfer in just one monomer. Domain rearrangements of this first monomer would then lead to an enhanced accessibility of the two neighboring monomers' $\mathrm{R}$ domains, perpetuating the activation through the hexamer. Notably, the side chain of $\operatorname{Ser}^{901}$ is situated only $5.4 \AA$ away from $\mathrm{Arg}^{900}$ of one adjacent monomer, allowing an R-R contact upon phosphorylation that could eventually sequester together all six untethered $\mathrm{R}$ domains. This could aid the activation process and stabilize the activated state. A similar interaction between inhibitory domains has been suggested in a recent cross-linking study on the plant proton pump AHA2 (40).

\section{Pma1 as a drug target}

A main incentive of determining a fungal $\mathrm{H}^{+}$-ATPase structure is to provide the basis for developing small-molecule inhibitors as novel 
A

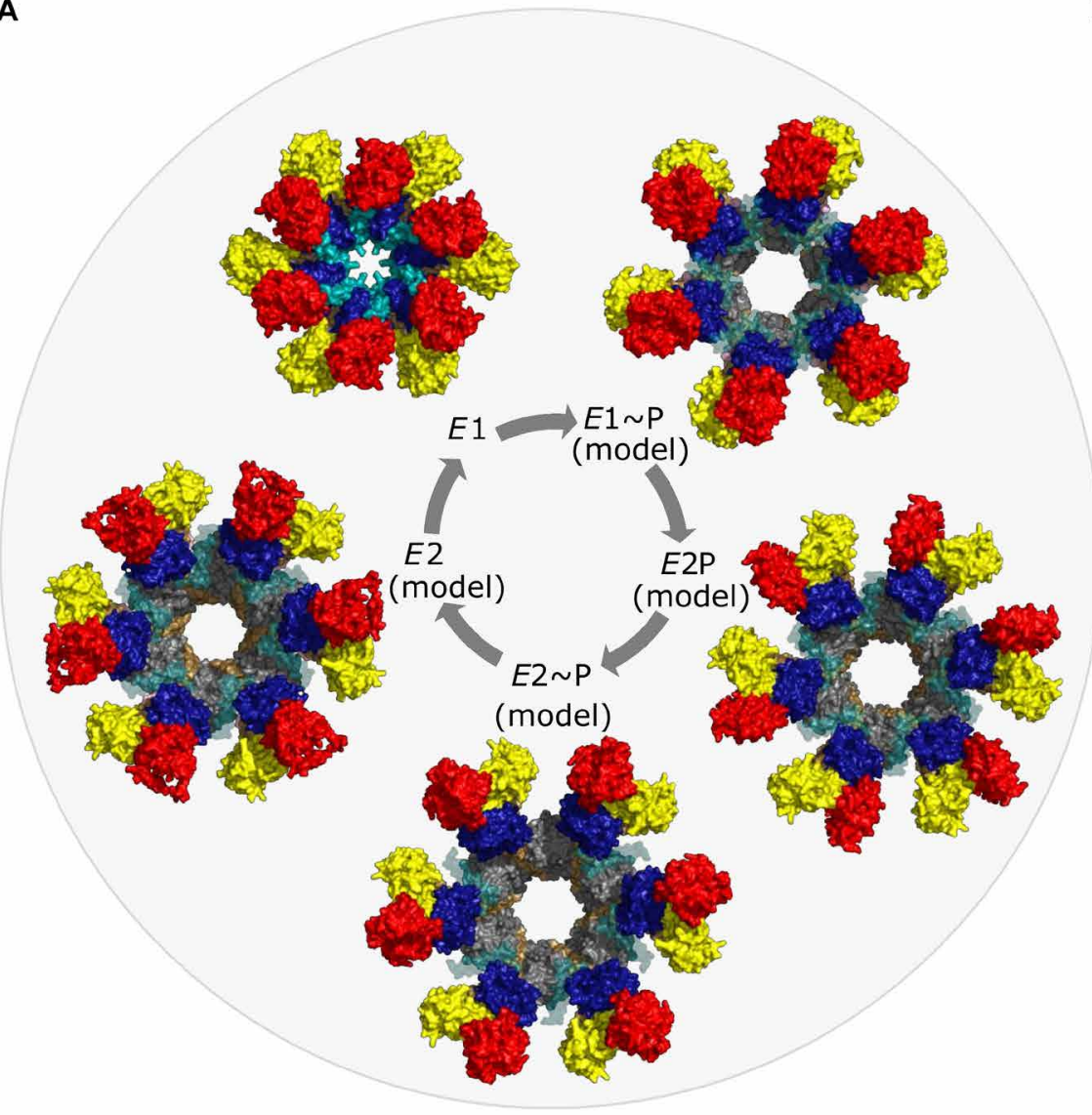

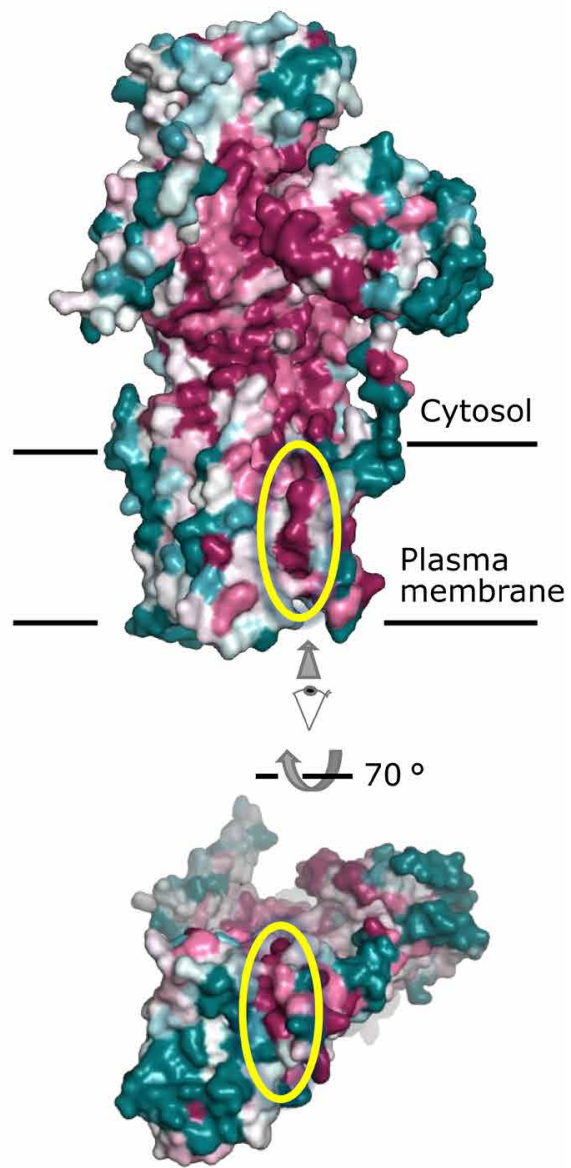

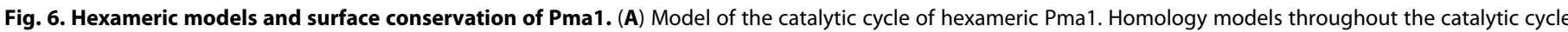

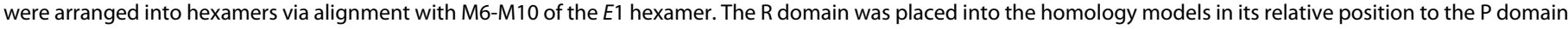

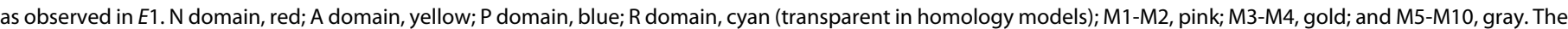

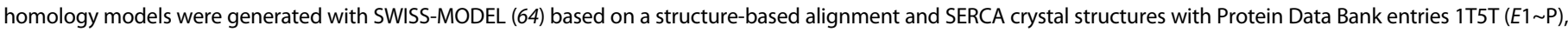

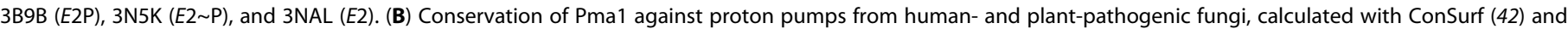

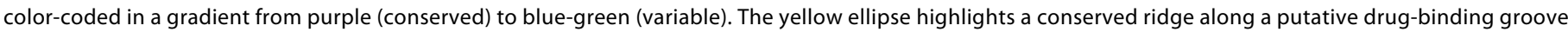
in the $\mathrm{M}$ domain, which extends into the extracytosolic side of Pma1.

antifungals. To explore the amenability of Pmal to specific smallmolecule drugs, we aligned the $N$. crassa Pmal primary sequence to that of the most important human- and plant-pathogenic fungi (table S6) and plotted the conservation score on the protein surface using the ConSurf Server (Fig. 6B) (42). Focusing on the transmembrane and extracellular regions, which would be least conserved relative to human P-type ATPases and most accessible by drugs, we identified a putative drug-binding pocket in a deep groove between M1, M3, and M4, along a ridge of highly conserved residues, and extending to the extracellular surface (Fig. 6B). To probe this groove's suitability for drug binding, we docked a set of nine recently reported novel Pmal inhibitors [tetrahydrocarbazoles (THCAs)] (43) into the Pmal structure, using the entire transmembrane and extracellular region of one monomer as search space (fig. S14). Out of the total 162 calculated docking poses, 21 poses both had a calculated binding affinity of $<-8.5 \mathrm{kcal} / \mathrm{mol}$ and had not been docked into the hexamer interface. Encouragingly, 18 of these 21 docking poses were located in the conserved groove described above (fig. S14).

\section{DISCUSSION}

The Pmal structure reveals that core elements of all known protonpumping proteins are conserved within the Pmal monomer: (i) a central proton acceptor/donor $\left(\mathrm{Asp}^{730}\right)$, (ii) a positively charged residue to control $\mathrm{p} K_{\mathrm{a}}$ changes of the proton acceptor/donor $\left(\mathrm{Arg}^{695}\right)$, and (iii) bound water molecules to facilitate rapid proton transport by Grotthus shuttling (44).

The properties of the Pmal hexamer reveal further important insights: The dependence of Pmal activity on anionic lipids can be rationalized by a preferential interaction site for these lipids at the monomer interface. The function of the anionic lipids is likely not limited to the mediation of hexamer contacts but could also be involved in the attraction of protons into the acceptor site. This is in line with our finding of a region of local membrane deformation and a concomitant clustering of cations. Local attractions between the lipid bilayer surface and the protein may also facilitate or accelerate conformational changes in the protein. Mutual adaptations between protein and membrane, as well as membrane deformations assisting cytosolic ion entry have already been suggested 
for SERCA $(17,29)$ and might be a common feature of P-type ATPase function.

We find no structural basis for cooperativity between the monomers in the hexamer, but the structure implies that autoinhibition is enhanced by -if not dependent on-a hexamer context. Activation by phosphorylation must occur sequentially because of steric hindrance and is probably accelerated by mutual sequestering of the released $\mathrm{R}$ domains. Further investigations on the structure-function relationship of glucose-activated Pmal, as well as on mutants unable to hexamerize, will clarify these issues. Furthermore, the putative inhibitor-binding groove suggested by our docking studies will need to be confirmed experimentally. Notably, most of the conserved residues around this groove are also conserved in the related plant proton pump (table S5), making this site a potentially more promising target for therapeutic applications than for plant protection.

\section{MATERIALS AND METHODS}

\section{Plasma membrane preparation}

Purification of Pmal was carried out from a native source, using a cell wall-less N. crassa strain [Fungal Genetics Stock Center (FGSC), strain \#4761]. Cells were grown for 24 hours at $30^{\circ} \mathrm{C}$ and $185 \mathrm{rpm}$ (Brunswick Innova 44) in Vogel's medium supplemented with 2\% $(\mathrm{w} / \mathrm{v})$ mannitol, $0.75 \%(\mathrm{w} / \mathrm{v})$ yeast extract (Difco), and $0.75 \%(\mathrm{w} / \mathrm{v})$ nutrient broth (Difco) using 2.5-liter Ultra Yield flasks with AirOtop lids. Plasma membrane preparation was carried out as described previously (46) with some adjustments to the original protocol. All centrifugation steps were conducted at $4^{\circ} \mathrm{C}$; cells and membranes were kept on ice unless stated otherwise. The cells were harvested by centrifugation $(15 \mathrm{~min}$ at $700 \mathrm{~g})$ and washed four times with ice-cold buffer A [ $50 \mathrm{mM}$ tris (pH 7.5), $10 \mathrm{mM} \mathrm{MgSO}_{4}$, and $250 \mathrm{mM}$ mannitol] (15 min, 700g) before they were agglutinated with concanavalin A $(0.5 \mathrm{mg} / \mathrm{ml})$ in buffer A for $10 \mathrm{~min}$ at room temperature (RT) and another $10 \mathrm{~min}$ on ice. Agglutinated cells were pelleted for $6 \mathrm{~min}$ at $200 \mathrm{~g}$ and washed once with ice-cold buffer A. Lysis was obtained by homogenizing the cells in ice-cold lysis buffer [10 mM tris ( $\mathrm{pH} 7.5)$, $1 \mathrm{mM} \mathrm{MgCl} 2,1 \mathrm{mM} \mathrm{CaCl}$, chymostatin $(1.5 \mu \mathrm{g} / \mathrm{ml})$, and deoxyribonuclease (DNase) I $(1 \mu \mathrm{g} / \mathrm{ml})]$ containing $1.2 \%$ sodium deoxycholate (DOC) using a $50-\mathrm{cm}^{3}$ glass homogenizer. After centrifugation (30 min, 14,000g), the membrane pellet was first washed with buffer B $[10 \mathrm{mM}$ tris (pH 7.5) and chymostatin $(1.5 \mu \mathrm{g} / \mathrm{ml})]$ containing $0.6 \%$ DOC and then with DOC-free buffer B. Dissociation of concanavalin A from the plasma membrane was achieved by incubation with $0.5 \mathrm{M}$ $\alpha$-methylmannoside in buffer B for $5 \mathrm{~min}$ at $30^{\circ} \mathrm{C}$. The dissociated membranes were diluted with ice-cold buffer $B$, pelleted $(30 \mathrm{~min}$, $14,000 \mathrm{~g}$ ), and washed once with ice-cold buffer B. After resuspension in ice-cold buffer $\mathrm{B}$, membranes were flash-frozen in liquid nitrogen and stored at $-80^{\circ} \mathrm{C}$ until protein purification.

\section{Detergent solubilization and protein purification}

Membranes were thawed in a RT water bath, spun down (30 min, $17,700 \mathrm{~g}, 4^{\circ} \mathrm{C}$ ), and washed twice [ $20 \mathrm{mM}$ Hepes $(\mathrm{pH} 7.2), 0.5 \mathrm{M} \mathrm{NaCl}$, and $1 \mathrm{mM}$ phenylmethylsulfonyl fluoride]. Solubilization was carried out for 1 hour at $12^{\circ}$ to $19^{\circ} \mathrm{C}$ with $2.25 \mathrm{mg} / \mathrm{ml}$ of the detergent DDM in Pmal solubilization buffer [10 mM tris (pH 7.5), $150 \mathrm{mM}$ $\mathrm{NaCl}, 1 \mathrm{mM} \mathrm{Na}$-ATP, $5 \mathrm{mM} \mathrm{MgSO}_{4}, 0.1 \mathrm{mM} \mathrm{Na}_{3} \mathrm{VO}_{4}$, and chymostatin $(2 \mu \mathrm{g} / \mathrm{ml})]$ using a total membrane protein concentration of $2 \mathrm{mg} / \mathrm{ml}$ determined by bicinchoninic acid assay. All further purification and centrifugation steps were performed at $4^{\circ} \mathrm{C}$. Unsolubilized membranes were removed by centrifugation ( $50 \mathrm{~min}, 17,700 \mathrm{~g}$ ), and the supernatant was filtered through a $0.45-\mu \mathrm{m}$ nylon membrane.

The protein solution was concentrated by pressure dialysis with a stirred cell (Amicon) and a $300-\mathrm{kDa}$ molecular weight cutoff (MWCO) filter membrane, diluted to a $\mathrm{NaCl}$ concentration of $\sim 40$ to $45 \mathrm{mM}$ [50 mM MES/tris (pH 7), $5 \mathrm{mM} \mathrm{MgSO}$, glycerol (200 g/liter), $1 \mathrm{mM}$ dithiothreitol (DTT), and $2 \mathrm{mM}$ EDTA], and loaded onto a Q HP anion exchange (AEX) column (GE Healthcare) equilibrated with dilution buffer containing DDM $(0.1 \mathrm{mg} / \mathrm{ml})$. The AEX column was washed with 20 column volumes (CV) of wash buffer $[50 \mathrm{mM}$ MES/tris (pH 7), $20 \mathrm{mM} \mathrm{KCl,} 5 \mathrm{mM} \mathrm{MgSO}_{4}, 1 \mathrm{mM}$ DTT, 2 mM EDTA, $\operatorname{DDM}(0.15 \mathrm{mg} / \mathrm{ml})$, and chymostatin $(2 \mu \mathrm{g} / \mathrm{ml})]$ and eluted with a linear gradient to $500 \mathrm{mM} \mathrm{KCl}$ over $20 \mathrm{CV}$. Peak elution fractions were pooled, diluted 1:10 [50 $\mathrm{mM}$ MES/tris (pH 6), $5 \mathrm{mM} \mathrm{MgSO}_{4}, 2 \mathrm{mM}$ EDTA, $1 \mathrm{mM} \mathrm{DTT}$, chymostatin $(2 \mu \mathrm{g} / \mathrm{ml})$, and DDM $(0.15 \mathrm{mg} / \mathrm{ml})]$, and concentrated to a volume of 1 to $2 \mathrm{ml}$ with Amicon Ultra centrifugal filters (100-kDa MWCO).

The concentrated sample was loaded onto a glycerol density gradient from 20 to $40 \%(\mathrm{w} / \mathrm{v})$ (5\% steps) and ultracentrifuged for 16 hours at 34,400g (Optima L-100 XP, Ti70 rotor, acceleration 2, no brake). The gradient was harvested in 1-ml fractions from bottom to top using a peristaltic pump with high-performance liquid chromatography tubing (inside diameter of $0.15 \mathrm{~mm}$ ). The purest fractions were pooled, diluted 1:10 in glycerol-free buffer, and concentrated to a volume of 0.5 to $1 \mathrm{ml}$. The remaining Pma1-containing fractions were also pooled, diluted 1:5, and concentrated to a volume of 0.5 to $1 \mathrm{ml}$. The concentrated samples were loaded onto two separate glycerol density gradients from 20 to $45 \%$ (w/v) (5\% steps), ultracentrifuged, and harvested as described above. The purest fractions of both gradients were pooled, concentrated, and loaded onto a Superose 6 Increase 10/300 (GE Healthcare) size exclusion chromatography (SEC) column equilibrated with SEC buffer [50 mM MES/tris ( $\mathrm{pH}$ 6.5), glycerol (200 g/liter), $50 \mathrm{mM} \mathrm{KCl}, 5 \mathrm{mM} \mathrm{MgSO}_{4}, 2 \mathrm{mM}$ EDTA, $1 \mathrm{mM}$ DTT, chymostatin $(2 \mu \mathrm{g} / \mathrm{ml})$, and DDM $(0.15 \mathrm{mg} / \mathrm{ml})]$. Peak fractions were evaluated by silver stain SDS-polyacrylamide gel electrophoresis and negative-stain EM.

\section{Negative-stain EM}

Samples were diluted to $0.015 \mathrm{mg} / \mathrm{ml}$ [determined with NanoDrop One and Abs $0.1 \%=1.044$ calculated by ProtParam (47)] with glycerolfree SEC buffer. Carbon-coated 400-mesh copper grids were glowdischarged ( $15 \mathrm{~mA}, 45 \mathrm{~s})$, incubated with $3 \mu \mathrm{l}$ of sample for $30 \mathrm{~s}$, and stained twice for $15 \mathrm{~s}$ with $3 \mu \mathrm{l}$ of $2 \%$ uranyl formate. Blotting after each incubation step was performed at a $45^{\circ}$ angle with Whatman filter paper (\#1). The grids were imaged with $120-\mathrm{kV}$ Tecnai Spirit (Thermo Fisher Scientific) at $\times 52,000$ magnification.

\section{Cryo-EM}

Suitable SEC elution fractions (see fig. S5) were pooled and concentrated to $40 \mu \mathrm{l}$ at $3 \mathrm{mg} / \mathrm{ml}$ with Amicon Ultra centrifugal filters $(100-\mathrm{kDa}$ MWCO). The buffer was exchanged to cryo-EM buffer $[30 \mathrm{mM}$ MES (pH 6.5), $1 \mathrm{mM} \mathrm{MgSO}, 1 \mathrm{mM}$ DTT, chymostatin $(2 \mu \mathrm{g} / \mathrm{ml})$, and DDM $(0.15 \mathrm{mg} / \mathrm{ml})]$ with a $0.5-\mathrm{ml}$ Zeba Spin column $(7 \mathrm{kDa})$ following the manufacturer's protocol. The eluted sample was concentrated to $3.7 \mathrm{mg} / \mathrm{ml}$ and incubated for 2 hours on ice with $1 \mathrm{mM}$ $\mathrm{ADP}$ and $0.6 \mathrm{mM} \mathrm{AlF}_{4}$ at a final protein concentration of $2 \mathrm{mg} / \mathrm{ml}$ $(\sim 20 \mu \mathrm{M})$. Cryo-EM grids were prepared by applying $3 \mu \mathrm{l}$ of sample to twice glow-discharged ( $15 \mathrm{~mA}, 45 \mathrm{~s}$ ) C-flat R2/2 400-mesh copper grids, which were blotted for $11 \mathrm{~s}\left(10^{\circ} \mathrm{C}, 70 \%\right.$ humidity, S\&S 595 
filter paper) and plunge-frozen in liquid ethane using Vitrobot Mark IV (Thermo Fisher Scientific). Cryo-EM grids were stored in liquid nitrogen until data were collected with 300-kV Titan Krios G3i (Thermo Fisher Scientific) equipped with a K3 direct electron detector (Gatan). Micrographs (2780) were recorded automatically in EPU (Thermo Fisher Scientific) in counting mode at $105,000 \times$ magnification with a calibrated pixel size of $0.837 \AA$. The defocus range was set from -1.3 to $-2.5 \mu \mathrm{m}$, and each micrograph was dosefractionated to 50 frames with a total exposure time of $3 \mathrm{~s}$, resulting in a total dose of $\sim 42 e^{-} / \AA^{2}$ (see table S1).

\section{Cryo-EM image analysis}

CryoSPARC (48) was used for particle picking and selection, while all further refinement steps were performed in Relion (49-51). After patch-based motion correction and contrast transfer function (CTF) estimation in cryoSPARC, particles were picked with the blob picker (180 A diameter) and manually curated, resulting in 483,742 particles. Four iterative rounds of $2 \mathrm{D}$ classification led to 95,525 particles, which were used for an ab initio reconstruction with three classes, resulting in two protein classes with 81,009 particles and one junk class. A homogeneous refinement gave an initial map of hexameric Pmal at 3.6- $\AA$ resolution (using C1 symmetry). The raw micrographs were then imported to Relion 3.0 and motioncorrected with MotionCor2 (52). CTF estimation was performed using Gctf (53) on non-dose-weighted aligned micrographs, and the cryoSPARC particles were reextracted using the coordinates from the homogeneous refinement. The following processing steps were performed in Relion 3.1 beta. An initial 3D refinement applying C6 symmetry resulted in a $3.79-\AA$ map. CTF refinement and anisotropic magnification estimation (54) improved the resolution to $3.53 \AA$, Bayesian polishing (55) to $3.42 \AA$ (map H1), and another CTF refinement to $3.28 \AA$ (map H2). The map was of high quality for the $\mathrm{M}$ and $\mathrm{P}$ domains and part of the $\mathrm{R}$ domain, but the $\mathrm{A}$ and $\mathrm{N}$ domains were less well resolved. Then, the hexamer particles from the map $\mathrm{H} 2$ were subjected to a 3D classification focused on the most rigid part of the hexamer ( $\mathrm{M}$ and $\mathrm{P}$ domains). The remaining 59,511 particles $(73.3 \%)$ were $3 \mathrm{D}$ refined (with a mask around the whole hexamer) to 3.28 - $\AA$ resolution (map H3). The map was further postprocessed with DeepEMhancer (56) (map H3-DE) and density-modified (model-free) using phenix.resolve_cryo_em (57) (H3-DM) to aid interpretation and visualization.

To further improve the resolution of the cytosolic $\mathrm{A}$ and $\mathrm{N}$ domains, the particles of map $\mathrm{H} 2$ were symmetry-expanded and a focused 3D classification was performed (50) using a monomer mask. The best monomer class appeared in $59.9 \%(293,999)$ of the expanded hexamer particles, which were then used for 3D refinement applying $\mathrm{C} 1$ symmetry and only local angular searches, to prevent larger particle rotations (50). The refinement was started with a mask encompassing the whole hexamer to prevent particle misalignment. From a later iteration, the refinement was continued using a mask that again covered the same monomer on which the $3 \mathrm{D}$ classification had been focused. Focused refinement improved the map resolution to $3.21 \AA$ (map $\mathrm{M}$ ) and improved the signal for the cytosolic A and $\mathrm{N}$ domains, allowing model building. Map $\mathrm{M}$ was further postprocessed with DeepEMhancer (map M-DE) and density-modified (model-free) with phenix.resolve_cryo_em (57) (map M-DM), which further aided model building, evaluation, and map visualization. A summary of the refinement process is depicted in fig. S2A.

\section{Model building and refinement}

The original and postprocessed versions of monomer map $\mathrm{M}$ were used for de novo model building in Coot (58), assisted by homology models (see below) and the Namdinator server (59). Amino acid residue assignment was based on clearly defined densities of bulky residues (Phe, Trp, Tyr, and Arg), the MgADP ligand, and $\mathrm{K}^{+}$ion that were added to the model based on the crystal structures of the plant proton pump $(16)$ and SERCA $(17,30)$. The final model was refined using phenix.real_space_refine (60) and the density-modified monomer map M-DM. To generate the hexameric model from the refined monomer, C6 symmetry was applied to expand the monomer model into the density-modified hexamer map H3 using UCSF Chimera (61). The geometry of both models was validated using MolProbity (62) implemented in the Phenix Comprehensive validation tool (63) (see table S1). Final models of the monomer and hexamer were submitted to the Protein Data Bank, PDB ID 7NXF and $7 \mathrm{NY} 1$, respectively.

\section{Homology modeling and internal cavities}

Homology models were generated with SWISS-MODEL (64) based on a structure-based alignment (65) and the SERCA crystal structures 1T5T (E1 P) (30), 3B9B (E2P) (66), 3N5K (E2 P) (67), and 3NAL (E2) (68). The SWISS-MODEL homology models were aligned to monomeric and hexameric Pma1 via M6-M10. Internal cavities of monomeric Pmal and homology models were detected with HOLLOW (69).

\section{Inhibitor docking}

The docking of a set of THCA Pmal inhibitors (43) into the structure of the Pmal monomer was done with AutoDock Vina (70). Nine THCA compounds were taken from a previous study (43), for which they had been built and energy-minimized in MAESTRO (Maestro, Schrödinger LLC, New York, NY, 2017) and prepared for docking in AutoDockTools, making all relevant bonds rotatable. Ligands were added in both their $\mathrm{R}$ and $\mathrm{S}$ configurations to a box encompassing the entire transmembrane domain of Pmal. Nine binding modes per configuration were analyzed, resulting in a total of 162 binding events of which 60 displayed affinities stronger than $-8.5 \mathrm{kcal} /$ mol. Thirty-nine binding events occurred at one of the monomer interfaces and were therefore excluded from further analysis. Three binding events took place within the proton entry funnel, and 18 within the exit funnel. The strongest binding, exceeding $-9 \mathrm{kcal} / \mathrm{mol}$, was observed for 10 events within the exit funnel. Residues involved in the putative binding of the respective THCA compounds $(6 \mathrm{~S}, 7 \mathrm{~S} / \mathrm{R}$, and 8R) were identified using LigPlot+ (see fig. S14) (71).

\section{Coarse-grained MD simulations}

The pentamer structure coordinates were converted to coarse-grained Martini representation using the martinize script (72). Their tertiary structures were constrained using the ElNeDyn elastic network between all chains (73) with a force constant of $500 \mathrm{~kJ} / \mathrm{mol}$ per $\mathrm{nm}^{2}$ and a cutoff of $0.9 \mathrm{~nm}$. The CG protein coordinates were then positioned in the center of a simulation box of size $23 \mathrm{~nm}$ by $23 \mathrm{~nm}$ by $23 \mathrm{~nm}$, with its principal transmembrane axis aligned parallel to the $z$ axis and embedded in a complex asymmetric membrane bilayer composed of 14 lipid species using the insane script (74). The membrane bilayer was built according to table $\mathrm{S6}$. To investigate the role of lipid saturation, we also set up simulations of pentameric Pma1 in a 50\% C16:0/18:2 PC (PIPC):50\% DIPC symmetric membrane. $\mathrm{NaCl}(0.15 \mathrm{M})$ was added to the solvated system. The Martini 
coarse-grained force field version 2.2 (72) was used for protein and version 2.0 for lipids. All the simulations were performed using GROMACS 2019.1 (75). The systems were energy-minimized using the steepest descents method, equilibrated for 50 ns using 20 -fs time steps in the isothermal-isobaric (NPT) ensemble at $323 \mathrm{~K}$ using the $\mathrm{V}$-rescale thermostat (76) and at 1 bar using a semi-isotropic Berendsen barostat (77). Five production simulations were run to $10 \mu$ s using the Parrinello-Rahman barostat (78). Data were analyzed using VMD (79), GROMACS tools (77), and in-house scripts. A weighted atomic density for $\mathrm{Na}^{+}$at each grid point was calculated using the VMD VolMap tool over $50 \mu$ s of simulation with default settings. Plots were made using MDAnalysis (80) and Microsoft Excel.

\section{Figure preparation}

Figures were prepared using VMD (79), ChimeraX (81), and PyMOL (82). The OPM database (45) was used to estimate the membrane position for Fig. 2C. EM maps shown in figures are M-DE (Fig. 1A and fig. S2D), M-DM (fig. S4), H3 (fig. S2B), and H3-DE (Fig. 1B and fig. S2C).

\section{SUPPLEMENTARY MATERIALS}

Supplementary material for this article is available at https://science.org/doi/10.1126/ sciadv.abj5255

View/request a protocol for this paper from Bio-protocol.

\section{REFERENCES AND NOTES}

1. M. Palmgren, $\mathrm{P}$. Morsomme, The plasma membrane $\mathrm{H}^{+}$-ATPase, a simple polypeptide with a long history. Yeast 36, 201-210 (2019).

2. C. L. Slayman, Electrical properties of Neurospora crassa. Effects of external cations on the intracellular potential. J. Gen. Physiol. 49, 69-92 (1965).

3. D. Seto-Young, B. Monk, A. B. Mason, D. S. Perlin, Exploring an antifungal target in the plasma membrane $\mathrm{H}^{+}$-ATPase of fungi. Biochim. Biophys. Acta 1326, 249-256 (1997).

4. M. Richardson, C. Lass-Flörl, Changing epidemiology of systemic fungal infections. Clin. Microbiol. Infect. 14 (suppl. 4), 5-24 (2008).

5. M. Auer, G. A. Scarborough, W. Kühlbrandt, Three-dimensional map of the plasma membrane $\mathrm{H}^{+}$-ATPase in the open conformation. Nature 392, 840-843 (1998).

6. M. Bagnat, A. Chang, K. Simons, Plasma membrane proton ATPase Pma1p requires raft association for surface delivery in yeast. Mol. Biol. Cell 12, 4129-4138 (2001).

7. S. Hippe, H. Lüth, A simple physical model for fungicide induced hexagonal clustering of intramembrane particles in the plasmalemma of Ustilago avenae. J. Theor. Biol. 121, 351-366 (1986).

8. P. Matile, H. Moor, K. Mühlethaler, Isolation and properties of the plasmalemma in yeast. Arch. Mikrobiol. 58, 201-211 (1967).

9. P. Eraso, F. Portillo, Molecular mechanism of regulation of yeast plasma membrane $\mathrm{H}^{+}$-ATPase by glucose. Interaction between domains and identification of new regulatory sites. J. Biol. Chem. 269, 10393-10399 (1994).

10. W. Kühlbrandt, J. Zeelen, J. Dietrich, Structure, mechanism, and regulation of the Neurospora plasma membrane $\mathrm{H}^{+}$-ATPase. Science 297, 1692-1696 (2002).

11. M. C. S. Lee, S. Hamamoto, R. Schekman, Ceramide biosynthesis is required for the formation of the oligomeric $\mathrm{H}^{+}$-ATPase Pma1p in the yeast endoplasmic reticulum. J. Biol. Chem. 277, 22395-22401 (2002).

12. B. Gaigg, B. Timischl, L. Corbino, R. Schneiter, Synthesis of sphingolipids with very long chain fatty acids but not ergosterol is required for routing of newly synthesized plasma membrane ATPase to the cell surface of yeast. J. Biol. Chem. 280, 22515-22522 (2005).

13. B. Gaigg, A. Toulmay, R. Schneiter, Very long-chain fatty acid-containing lipids rather than sphingolipids per se are required for raft association and stable surface transport of newly synthesized plasma membrane ATPase in yeast. J. Biol. Chem. 281, 34135-34145 (2006).

14. R. Addison, G. A. Scarborough, Solubilization and purification of the Neurospora plasma membrane $\mathrm{H}^{+}$-ATPase. J. Biol. Chem. 256, 13165-13171 (1981).

15. G. A. Scarborough, Properties of the Neurospora crassa plasma membrane ATPase. Arch. Biochem. Biophys. 180, 384-393 (1977).

16. B. P. Pedersen, M. J. Buch-Pedersen, J. Preben Morth, M. G. Palmgren, P. Nissen, Crystal structure of the plasma membrane proton pump. Nature 450, 1111-1114 (2007).

17. A.-M. L. Winther, M. Bublitz, J. L. Karlsen, J. V. Møller, J. B. Hansen, P. Nissen, The sarcolipin-bound calcium pump stabilizes calcium sites exposed to the cytoplasm. Nature 495, 265-269 (2013).
18. J. T. Pedersen, J. Falhof, K. Ekberg, M. J. Buch-Pedersen, M. Palmgren, Metal fluoride inhibition of a P-type $\mathrm{H}^{+}$pump. J. Biol. Chem. 290, 20396-20406 (2015).

19. M. R. Sussman, J. E. Strickler, K. M. Hager, C. W. Slayman, Location of a dicyclohexylcarbodiimide-reactive glutamate residue in the Neurospora crassa plasma membrane H+-ATPase. J. Biol. Chem. 262, 4569-4573 (1987).

20. M. J. Buch-Pedersen, M. G. Palmgren, Conserved Asp684 in transmembrane segment M6 of the plant plasma membrane P-type proton pump AHA2 is a molecular determinant of proton translocation. J. Biol. Chem. 278, 17845-17851 (2003).

21. M. Miranda, J. P. Pardo, V. V. Petrov, Structure-function relationships in membrane segment 6 of the yeast plasma-membrane Pma1 $\mathrm{H}^{+}$-ATPase. Biochim. Biophys. Acta 1808, 1781-1789 (2011).

22. D. Focht, T. I. Croll, B. P. Pedersen, P. Nissen, Improved model of proton pump crystal structure obtained by interactive molecular dynamics flexible fitting expands the mechanistic model for proton translocation in P-Type ATPases. Front. Physiol. 8, (2017).

23. V. V. Petrov, K. P. Padmanabha, R. K. Nakamoto, K. E. Allen, C. W. Slayman, Functional role of charged residues in the transmembrane segments of the yeast plasma membrane $\mathrm{H}^{+}$-ATPase. J. Biol. Chem. 275, 15709-15716 (2000).

24. K. J. Friedman, D. Glick, Role of lipids in the Neurospora crassa membrane: III. Lipid composition and phase transition properties of the plasma membrane, and its components. J. Membr. Biol. 54, 183-190 (1980).

25. L. R. Aaronson, C. E. Martin, Temperature-induced modifications of glycosphingolipids in plasma membranes of Neurospora crassa. Biochim. Biophys. Acta Biomembr. 735, 252-258 (1983).

26. J. S. van't Klooster, T.-Y. Cheng, H. R. Sikkema, A. Jeucken, B. Moodt, B. Poolman, Periprotein lipidomes of Saccharomyces cerevisiae provide a flexible environment for conformational changes of membrane proteins. eLife 9, e57003 (2020).

27. S. Matsunaga, T. Yamada, T. Kobayashi, M. Kawai, Scanning tunneling microscope observation of the phosphatidylserine domains in the phosphatidylcholine monolayer. Langmuir 31, 5449-5455 (2015).

28. T. Meier, U. Matthey, F. Henzen, P. Dimroth, D. J. Müller, The central plug in the reconstituted undecameric c cylinder of a bacterial ATP synthase consists of phospholipids. FEBS Lett. 505, 353-356 (2001).

29. Y. Sonntag, M. Musgaard, C. Olesen, B. Schiøtt, J. V. Møller, P. Nissen, Mutual adaptation of a membrane protein and its lipid bilayer during conformational changes. Nat. Commun. 2, 304 (2011).

30. T. L.-M. Sørensen, J. V. Møller, P. Nissen, Phosphoryl transfer and calcium ion occlusion in the calcium pump. Science 304, 1672-1675 (2004).

31. M. G. G. Geurts, J. D. Clausen, B. Arnou, C. Montigny, G. Lenoir, R. A. Corey, C. Jaxel, J. V. Møller, P. Nissen, J. P. Andersen, M. le Maire, M. Bublitz, The SERCA residue Glu340 mediates interdomain communication that guides $\mathrm{Ca}^{2+}$ transport. Proc. Natl. Acad. Sci. U.S.A. 117, 31114-31122 (2020)

32. C. R. Søndergaard, M. H. M. Olsson, M. Rostkowski, J. H. Jensen, Improved treatment of ligands and coupling effects in empirical calculation and rationalization of pKa values. J. Chem. Theory Comput. 7, 2284-2295 (2011).

33. M. H. M. Olsson, C. R. Søndergaard, M. Rostkowski, J. H. Jensen, PROPKA3: Consistent treatment of internal and surface residues in empirical pKa predictions. J. Chem. Theory Comput. 7, 525-537 (2011).

34. P. Eraso, M. J. Mazón, F. Portillo, Yeast protein kinase Ptk2 localizes at the plasma membrane and phosphorylates in vitro the $\mathrm{C}$-terminal peptide of the $\mathrm{H}^{+}$-ATPase. Biochim. Biophys. Acta 1758, 164-170 (2006).

35. S. Lecchi, C. J. Nelson, K. E. Allen, D. L. Swaney, K. L. Thompson, J. Coon, M. R. Sussman, C. W. Slayman, Tandem phosphorylation of Ser-911 and Thr-912 at the $C$ terminus of yeast plasma membrane $\mathrm{H}^{+}$-ATPase leads to glucose-dependent activation. J. Biol. Chem. 282, 35471-35481 (2007)

36. S. Lecchi, K. E. Allen, J. P. Pardo, A. B. Mason, C. W. Slayman, Conformational changes of yeast plasma membrane $\mathrm{H}^{+}$-ATPase during activation by glucose: Role of threonine-912 in the carboxy-terminal tail. Biochemistry 44, 16624-16632 (2005).

37. C. C. Chadwick, E. Goormaghtigh, G. A. Scarborough, A hexameric form of the Neurospora crassa plasma membrane $\mathrm{H}^{+}$-ATPase. Arch. Biochem. Biophys. 252, 348-356 (1987).

38. E. Goormaghtigh, C. Chadwick, G. A. Scarborough, Monomers of the Neurospora plasma membrane $\mathrm{H}^{+}$-ATPase catalyze efficient proton translocation. J. Biol. Chem. 261, 7466-7471 (1986).

39. K. Ekberg, B. P. Pedersen, D. M. Sørensen, A. K. Nielsen, B. Veierskov, P. Nissen, M. G. Palmgren, M. J. Buch-Pedersen, Structural identification of cation binding pockets in the plasma membrane proton pump. Proc. Natl. Acad. Sci. U.S.A. 107, 21400-21405 (2010).

40. T. T. Nguyen, M. R. Blackburn, M. R. Sussman, Intermolecular and intramolecular interactions of the arabidopsis plasma membrane proton pump revealed using a mass spectrometry cleavable cross-linker. Biochemistry 59, 2210-2225 (2020).

41. B. J. Bowman, Kinetic evidence for interacting active sites in the Neurospora crassa plasma membrane ATPase. J. Biol. Chem. 258, 13002-13007 (1983). 
42. H. Ashkenazy, S. Abadi, E. Martz, O. Chay, I. Mayrose, T. Pupko, N. Ben-Tal, ConSurf 2016 An improved methodology to estimate and visualize evolutionary conservation in macromolecules. Nucleic Acids Res. 44, W344-W350 (2016).

43. M. Bublitz, L. Kjellerup, K. O'Hanlon Cohrt, S. Gordon, A. L. Mortensen, J. D. Clausen, T. D. Pallin, J. B. Hansen, A. T. Fuglsang, W. Dalby-Brown, A.-M. L. Winther, Tetrahydrocarbazoles are a novel class of potent P-type ATPase inhibitors with antifungal activity. PLOS ONE 13, e0188620 (2018).

44. M. J. Buch-Pedersen, B. P. Pedersen, B. Veierskov, P. Nissen, M. G. Palmgren, Protons and how they are transported by proton pumps. Pflugers Arch. 457, 573-579 (2009).

45. M. A. Lomize, I. D. Pogozheva, H. Joo, H. I. Mosberg, A. L. Lomize, OPM database and PPM web server: Resources for positioning of proteins in membranes. Nucleic Acids Res. $\mathbf{4 0}$ D370-D376 (2012).

46. G. A. Scarborough, [43] Large-scale purification of plasma membrane $\mathrm{H}^{+}$-ATPase from a cell wall-less mutant of Neurospora crassa. Methods Enzymol. 157, 574-579 (1988).

47. E. Gasteiger, C. Hoogland, A. Gattiker, S. Duvaud, M. R. Wilkins, R. D. Appel, A. Bairoch, Protein identification and analysis tools on the ExPASy server, in The Proteomics Protocols Handbook, J. M. Walker, Ed. (Humana Press, 2005), pp. 571-607.

48. A. Punjani, J. L. Rubinstein, D. J. Fleet, M. A. Brubaker, cryoSPARC: Algorithms for rapid unsupervised cryo-EM structure determination. Nat. Methods 14, 290-296 (2017)

49. S. H. W. Scheres, RELION: Implementation of a Bayesian approach to cryo-EM structure determination. J. Struct. Biol. 180, 519-530 (2012).

50. S. H. W. Scheres, Processing of structurally heterogeneous Cryo-EM data in RELION. Methods Enzymol. 579, 125-157 (2016).

51. J. Zivanov, T. Nakane, B. O. Forsberg, D. Kimanius, W. J. H. Hegen, E. Lindahl, S. H. W. Scheres, New tools for automated high-resolution cryo-EM structure determination in RELION-3. eLife 7, e42166 (2018).

52. S. Q. Zheng, E. Palovcak, J.-P. Armache, K. A. Verba, Y. Cheng, D. A. Agard, MotionCor2: Anisotropic correction of beam-induced motion for improved cryo-electron microscopy. Nat. Methods 14, 331-332 (2017).

53. K. Zhang, Gctf: Real-time CTF determination and correction. J. Struct. Biol. 193, 1-12 (2016).

54. J. Zivanov, T. Nakane, S. H. W. Scheres, Estimation of high-order aberrations and anisotropic magnification from cryo-EM data sets in RELION-3.1. IUCrJ 7, 253-267 (2020).

55. J. Zivanov, T. Nakane, S. H. W. Scheres, A Bayesian approach to beam-induced motion correction in cryo-EM single-particle analysis. IUCrJ 6, 5-17 (2019).

56. R. Sanchez-Garcia, J. Gomez-Blanco, A. Cuervo, J. M. Carazo, C. O. S. Sorzano, J. Vargas, DeepEMhancer: A deep learning solution for cryo-EM volume post-processing. Commun. Biol. 15, 874 (2021).

57. T. C. Terwilliger, S. J. Ludtke, R. J. Read, P. D. Adams, P. V. Afonine, Improvement of cryo-EM maps by density modification. Nat. Methods 17, 923-927 (2020).

58. A. Casañal, B. Lohkamp, P. Emsley, Current developments in Coot for macromolecular model building of Electron Cryo-microscopy and Crystallographic Data. Protein Sci. 29, 1069-1078 (2020).

59. R. T. Kidmose, J. Juhl, P. Nissen, T. Boesen, J. L. Karlsen, B. P. Pedersen, NamdinatorAutomatic molecular dynamics flexible fitting of structural models into cryo-EM and crystallography experimental maps. IUCrJ 6, 526-531 (2019).

60. P. V. Afonine, B. K. Poon, R. J. Read, O. V. Sobolev, T. C. Terwilliger, A. Urzhumtsev, P. D. Adams, Real-space refinement in PHENIX for cryo-EM and crystallography Acta Crystallogr. D Struct. Biol. 74, 531-544 (2018).

61. E. F. Pettersen, T. D. Goddard, C. C. Huang, G. S. Couch, D. M. Greenblatt, E. C. Meng T. E. Ferrin, UCSF Chimera-A visualization system for exploratory research and analysis. J. Comput. Chem. 25, 1605-1612 (2004).

62. C. J. Williams, J. J. Headd, N. W. Moriarty, M. G. Prisant, L. L. Videau, L. N. Deis, V. Verma, D. A. Keedy, B. J. Hintze, V. B. Chen, S. Jain, S. M. Lewis, W. B. Arendall 3rd, J. Snoeyink, P. D. Adams, S. C. Lovell, J. S. Richardson, D. C. Richardson, MolProbity: More and better reference data for improved all-atom structure validation. Protein Sci. 27, 293-315 (2018).

63. D. Liebschner, P. V. Afonine, M. L. Baker, G. Bunkóczi, V. B. Chen, T. I. Croll, B. Hintze, L. W. Hung, S. Jain, A. J. McCoy, N. W. Moriarty, R. D. Oeffner, B. K. Poon, M. G. Prisant, R. R. Read, J. S. Richardson, D. C. Richardson, M. D. Sammito, O. V. Sobolev,

D. H. Stockwell, T. C. Terwilliger, A. G. Urzhumtsev, L. L. Videau, C. J. Williams, P. D. Adams, Macromolecular structure determination using X-rays, neutrons and electrons: Recent developments in Phenix. Acta Crystallogr. D Struct. Biol. 75, 861-877 (2019).

64. M. Biasini, S. Bienert, A. Waterhouse, K. Arnold, G. Studer, T. Schmidt, F. Kiefer, T. G. Cassarinno, M. Bertoni, L. Bordoli, T. Schwede, SWISS-MODEL: Modelling protein tertiary and quaternary structure using evolutionary information. Nucleic Acids Res. 42, W252-W258 (2014)

65. D. R. Caffrey, P. H. Dana, V. Mathur, M. Ocano, E.-J. Hong, Y. E. Wang, S. Somaroo, B. E. Caffrey, S. Potluri, E. S. Huang, PFAAT version 2.0: A tool for editing, annotating, and analyzing multiple sequence alignments. BMC Bioinformatics 8, 381 (2007).

66. C. Olesen, M. Picard, A.-M. L. Winther, C. Gyrup, J. P. Morth, C. Oxvig, J. V. Møller, P. Nissen The structural basis of calcium transport by the calcium pump. Nature 450, 1036-1042 (2007).
67. M. Bublitz, M. Musgaard, H. Poulsen, L. Thøgersen, C. Olesen, B. Schiøtt, J. P. Morth, J. V. Møller, P. Nissen, lon pathways in the sarcoplasmic reticulum $\mathrm{Ca}^{2+}$-ATPase. J. Biol. Chem. 288, 10759-10765 (2013).

68. A.-M. L. Winther, H. Liu, Y. Sonntag, C. Olesen, M. le Maire, H. Sohoel, C.-E. Olsen, S. B. Christensen, P. Nissen, J. V. Møller, Critical roles of hydrophobicity and orientation of side chains for inactivation of sarcoplasmic reticulum $\mathrm{Ca}^{2+}$-ATPase with thapsigargin and thapsigargin analogs. J. Biol. Chem. 285, 28883-28892 (2010).

69. B. K. Ho, F. Gruswitz, HOLLOW: Generating accurate representations of channel and interior surfaces in molecular structures. BMC Struct. Biol. 8, 49 (2008).

70. O. Trott, A. J. Olson, AutoDock Vina: Improving the speed and accuracy of docking with a new scoring function, efficient optimization, and multithreading. J. Comput. Chem 31, 455-461 (2010)

71. R. A. Laskowski, M. B. Swindells, LigPlot+: Multiple ligand-protein interaction diagrams for drug discovery. J. Chem. Inf. Model. 51, 2778-2786 (2011).

72. D. H. de Jong, G. Singh, W. F. D. Bennett, C. Arnarez, T. A. Wassenaar, L. V. Schäfer, X. Periole D. P. Tieleman, S. J. Marrink, Improved parameters for the martini coarse-grained protein force field. J. Chem. Theory Comput. 9, 687-697 (2013).

73. X. Periole, M. Cavalli, S.-J. Marrink, M. A. Ceruso, Combining an elastic network with a coarse-grained molecular force field: Structure, dynamics, and intermolecular recognition. J. Chem. Theory Comput. 5, 2531-2543 (2009).

74. T. A. Wassenaar, H. I. Ingólfsson, R. A. Böckmann, D. P. Tieleman, S. J. Marrink, Computational lipidomics with insane: A versatile tool for generating custom membranes for molecular simulations. J. Chem. Theory Comput. 11, 2144-2155 (2015).

75. M. J. Abraham, T. Murtola, R. Schulz, S. Páll, J. C. Smith, B. Hess, E. Lindahl, GROMACS: High performance molecular simulations through multi-level parallelism from laptops to supercomputers. SoftwareX 1-2, 19-25 (2015)

76. G. Bussi, D. Donadio, M. Parrinello, Canonical sampling through velocity-rescaling. J. Chem. Phys. 126, 014101 (2007).

77. H. J. C. Berendsen, D. van der Spoel, R. van Drunen, GROMACS: A message-passing parallel molecular dynamics implementation. Comput. Phys. Commun. 91, 43-56 (1995).

78. M. Parrinello, A. Rahman, Polymorphic transitions in single crystals: A new molecular dynamics method. J. Appl. Phys. 52, 7182-7190 (1981).

79. W. Humphrey, A. Dalke, K. Schulten, VMD: Visual molecular dynamics. J. Mol. Graph. 14, 33-38 (1996)

80. N. Michaud-Agrawal, E. J. Denning, T. B. Woolf, O. Beckstein, MDAnalysis: A toolkit for the analysis of molecular dynamics simulations. J. Comput. Chem. 32, 2319-2327 (2011).

81. E. F. Pettersen, T. D. Goddard, C. C. Huang, E. C. Meng, G. S. Couch, T. I. Croll, J. H. Morria T. E. Ferrin, UCSF ChimeraX: Structure visualization for researchers, educators, and developers. Protein Sci. 30, 70-82 (2021)

82. W. Delano, The PyMOL Molecular Graphics System (2002); www.pymol.org.

Acknowledgments: We thank P. Nissen, N. Düring Drachmann, D. Focht, C. Grønberg, and M. Mazzorana for contributions to initial project design and purification strategy, and R. Henderson for help with establishing suitable cryo-EM conditions. We are grateful to S. Kaltwasser, S. Prinz, S. Welsch, and J. Demmer for technical assistance and help with data collection and analysis. We thank J. Vonck for help evaluating preliminary cryo-EM data collected for the project and G. Hummer for constructive discussions on simulation data analysis. Funding: This work is funded by the Academy of Medical Sciences Springboard Award (to M.B. and S.H.), John Fell Fund 152/059 (to M.B.), E P A Cephalosporin Fund CF346 (to M.B. and S.H.), FEBS Short-Term Fellowship (to S.H.), ERASMUS+ Staff Training Mobility program (to S.H.), Biochemical Society General Travel Grant (to S.H.), Wellcome Trust Graduate programme in Cellular Structural Biology 220063/Z/20/Z (to M.M.G.G.), and the Max Planck Society. This research was funded in whole, or in part, by the Wellcome Trust [220063/Z/20/Z]. For the purpose of Open Access, the author has applied a CC BY public copyright license to any Author Accepted Manuscript version arising from this submission. Author contributions: Conceptualization: M.B. Methodology: S.H. Investigation: S.H., M.M.G.G., M.B., and D.J.M. Formal analysis: M.B., S.H., M.M.G.G., B.J.M., and R.A.C. Visualization: S.H. and M.M.G.G. Resources: W.K. Supervision: M.B. Funding acquisition: M.B., S.H., and W.K. Writingoriginal draft: M.B. Writing—review and editing: M.B., S.H., M.M.G.G., R.A.C., B.J.M., and W.K. Competing interests: The authors declare that they have no financial or other competing interests. Data and materials availability: All data needed to evaluate the conclusions in the paper are present in the paper and/or the Supplementary Materials. Coordinates and EM maps have been deposited in the Protein Data Bank and EM Data Bank with the following accession numbers: monomer, 7NXF and EMD-12638; hexamer, 7NY1 and EMD-12644.

\section{Submitted 20 May 2021}

Accepted 22 September 2021

Published 10 November 2021

10.1126/sciadv.abj5255 


\section{ScienceAdvances}

\section{Structure of the hexameric fungal plasma membrane proton pump in its autoinhibited state}

Sabine HeitMaxwell M. G. GeurtsBonnie J. MurphyRobin A. CoreyDeryck J. MillsWerner KühlbrandtMaike Bublitz

Sci. Adv., 7 (46), eabj5255. • DOI: 10.1126/sciadv.abj5255

View the article online

https://www.science.org/doi/10.1126/sciadv.abj5255

Permissions

https://www.science.org/help/reprints-and-permissions 